Dai Tamaki

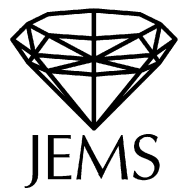

\title{
The Salvetti complex and the little cubes
}

Received September 11, 2008 and in revised form September 23, 2009

\begin{abstract}
For a real central arrangement $\mathcal{A}$, Salvetti introduced a construction of a finite complex $\operatorname{Sal}(\mathcal{A})$ which is homotopy equivalent to the complement of the complexified arrangement in [Sal87]. For the braid arrangement $\mathcal{A}_{k-1}$, the Salvetti complex $\operatorname{Sal}\left(\mathcal{A}_{k-1}\right)$ serves as a good combinatorial model for the homotopy type of the configuration space $F(\mathbb{C}, k)$ of $k$ points in $\mathbb{C}$, which is homotopy equivalent to the space $\mathcal{C}_{2}(k)$ of $k$ little 2-cubes. Motivated by the importance of little cubes in homotopy theory, especially in the study of iterated loop spaces, we study how the combinatorial structure of the Salvetti complexes of the braid arrangements is related to homotopytheoretic properties of iterated loop spaces.

We prove that the skeletal filtrations on the Salvetti complexes of the braid arrangements give rise to the cobar-type Eilenberg-Moore spectral sequence converging to the homology of $\Omega^{2} \Sigma^{2} X$. We also construct a new spectral sequence that computes the homology of $\Omega^{\ell} \Sigma^{\ell} X$ for $\ell>2$ by using a higher order analogue of the Salvetti complex. The $E^{1}$-term of the spectral sequence is described in terms of the homology of $X$. The spectral sequence is different from known spectral sequences that compute the homology of iterated loop spaces, such as the Eilenberg-Moore spectral sequence and the spectral sequence studied by Ahearn and Kuhn in [AK02].
\end{abstract}

\section{Introduction}

The aim of this article is to reveal an unexpected connection between the combinatorics of braid arrangements and homological properties of double (or more highly iterated) loop spaces.

\subsection{Homology of loop spaces}

In order to bridge different terminologies, notations, and interests in these two subjects, hyperplane arrangements and homology of loop spaces, let us first overview the difficulties in computing the homology of loop spaces.

Given a based space $Z$ and a homology theory $h_{*}(-)$, the homology $h_{*}(\Omega Z)$ of the loop space of $Z$ is a functor of $Z$. It would be nice if we could describe $h_{*}(\Omega Z)$ in terms of $h_{*}(Z)$. In general, we cannot expect such a nice situation, but we do have a spectral sequence whose $E^{2}$-term is a functor of $h_{*}(Z)$. Let us briefly recall the construction.

D. Tamaki: Department of Mathematical Sciences, Shinshu University, Matsumoto, 390-8621, Japan; e-mail: rivulus@math.shinshu-u.ac.jp 
Given a diagram of spaces

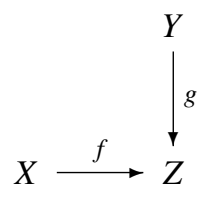

we can construct a cosimplicial space $\Omega^{\mathrm{geo}}(f, g)$, called the geometric cobar construction, as follows. The $k$-th space of $\Omega^{\text {geo }}(f, g)$ is given by

$$
\Omega^{\text {geo }}(f, g)^{k}=X \times Z^{k} \times Y
$$

and the structure maps

$$
d^{i}: \Omega^{\mathrm{geo}}(f, g)^{k} \longrightarrow \Omega^{\mathrm{geo}}(f, g)^{k+1}, \quad s^{i}: \Omega^{\mathrm{geo}}(f, g)^{k} \longrightarrow \Omega^{\mathrm{geo}}(f, g)^{k-1}
$$

are essentially given by diagonals, projections, $f$, and $g$. The total space of this cosimplicial space, $\operatorname{Tot}\left(\Omega^{\text {geo }}(f, g)\right)$, is known to be homeomorphic to the homotopy pullback of the diagram (1),

$$
\operatorname{Tot}\left(\Omega^{\mathrm{geo}}(f, g)\right) \cong \operatorname{holim}(X \stackrel{f}{\rightarrow} Z \stackrel{g}{\leftarrow} Y) .
$$

When $X=Y=*$, we obtain a cosimplicial model for the loop space of $Z$,

$$
\operatorname{Tot}(\Omega(*, *)) \cong \operatorname{holim}(* \stackrel{*}{\rightarrow} Z \stackrel{*}{\leftarrow} *)=\Omega Z \text {. }
$$

Rector [Rec70] used the cosimplicial model $\Omega^{\text {geo }}(f, g)$ to reformulate the construction of a spectral sequence originally obtained by Eilenberg and Moore [EM66a, EM66b].

When $h_{*}(-)$ is a multiplicative homology theory satisfying the strong form of the Künneth formula

$$
h_{*}(A \times B) \cong h_{*}(A) \otimes_{h_{*}} h_{*}(B),
$$

the $E^{2}$-term of the spectral sequence for the diagram (1) can be written as the homology of the algebraic cobar construction,

$$
E^{2} \cong H_{*}\left(\Omega^{\operatorname{alg}}\left(f_{*}, g_{*}\right)\right)
$$

where $\Omega^{\text {alg }}\left(f_{*}, g_{*}\right)$ is a negatively graded chain complex whose $(-k)$-th term is given by

$$
\Omega^{\mathrm{alg}}\left(f_{*}, g_{*}\right)_{-k}=h_{*}(X) \otimes_{h_{*}} h_{*}(Z)^{\otimes k} \otimes_{h_{*}} h_{*}(Y)
$$

and the boundaries are essentially given by the coalgebra structure on $h_{*}(Z)$ and the induced maps $f_{*}$ and $g_{*}$.

The homology of the cobar construction $\Omega^{\mathrm{alg}}\left(f_{*}, g_{*}\right)$ is isomorphic to the derived functor $\operatorname{Cotor}^{h_{*}(Z)}\left(h_{*}(X), h_{*}(Y)\right)$ of the cotensor product functor over the coalgebra $h_{*}(Z)$. Thus we obtain a spectral sequence with

$$
E^{2} \cong \operatorname{Cotor}^{h_{*}(Z)}\left(h_{*}(X), h_{*}(Y)\right) .
$$


When $h_{*}(-)$ is the ordinary homology theory with coefficients in a field $k$, the spectral sequence is known to converge to the homology of $\operatorname{Tot}\left(\Omega^{\text {geo }}(f, g)\right)$ under certain conditions. In particular, we have a spectral sequence

$$
E^{2} \cong \operatorname{Cotor}^{H_{*}(Z ; k)}(k, k) \Rightarrow H_{*}(\Omega Z ; k)
$$

when $Z$ is simply connected.

For other homology theories, especially nonconnective ones, however, the behavior of the spectral sequence could be disastrous and the $E^{\infty}$-term might not have any relation to the homology of $\operatorname{Tot}\left(\Omega^{\text {geo }}(f, g)\right)$ at all. For example, when $Z$ is the Eilenberg-Mac Lane space of $(\mathbb{Z} / p \mathbb{Z}, 2)$-type,

$$
Z=K(\mathbb{Z} / p \mathbb{Z}, 2)
$$

and $h_{*}(-)$ is the $\bmod p K$-theory,

$$
h_{*}(-)=K(-; \mathbb{Z} / p \mathbb{Z}),
$$

the $E^{2}$-term, and hence the $E^{\infty}$-term is trivial but $h_{*}(\Omega Z)=h_{*}(K(\mathbb{Z} / p \mathbb{Z}, 1))$ is known to be nontrivial. The spectral sequence does not give us any information on $h_{*}(\Omega Z)$.

There have been several attempts to find conditions under which the Eilenberg-Moore spectral sequence can be used to compute the homology of $\Omega Z$,

$$
E^{2} \cong \operatorname{Cotor}^{h_{*}(Z)}\left(h_{*}, h_{*}\right) \Rightarrow h_{*}(\Omega Z) .
$$

We still do not know a complete answer, but partial answers are known. One of such answers was given by the author. It is proved in [Tam94] that, when $Z=\Omega^{\ell-1} \Sigma^{\ell} X$, there is a spectral sequence with

$$
E^{2} \cong \operatorname{Cotor}^{h_{*}\left(\Omega^{\ell-1} \Sigma^{\ell} X\right)}\left(h_{*}, h_{*}\right)
$$

which splits into a direct sum of small spectral sequences each of which strongly converges to the corresponding summand in the decomposition

$$
\tilde{h}_{*}\left(\Omega^{\ell} \Sigma^{\ell} X\right) \cong \bigoplus_{k} \tilde{h}_{*}\left(\mathcal{C}_{\ell}(k)_{+} \wedge_{\Sigma_{k}} X^{\wedge k}\right) .
$$

This is the decomposition induced from the famous stable splitting due to Snaith [Sna74],

$$
\Sigma^{\infty} \Omega^{\ell} \Sigma^{\ell} X \simeq \Sigma^{\infty}\left(\bigvee_{k} \mathcal{C}_{\ell}(k)_{+} \wedge \Sigma_{k} X^{\wedge k}\right)
$$

Thus we do have a spectral sequence that computes $h_{*}(\Omega Z)$ in this case. It was also proved by the author [Tam07] that the spectral sequence (2) is isomorphic to the Eilenberg-Moore spectral sequence.

The space $\mathcal{C}_{\ell}(k)$ appearing in the direct sum decomposition (3) is the space of little cubes studied by May in [May72]. The author constructed the spectral sequence (2) by defining a filtration on each $\mathcal{C}_{\ell}(k)$,

$\emptyset=F_{-k-1} \mathcal{C}_{\ell}(k) \subset F_{-k} \mathcal{C}_{\ell}(k) \subset \cdots \subset F_{-2} \mathcal{C}_{\ell}(k) \subset F_{-1} \mathcal{C}_{\ell}(k)=F_{0} \mathcal{C}_{\ell}(k)=\mathcal{C}_{\ell}(k)$,

which is a completely different method from the cosimplicial construction of the Eilenberg-Moore spectral sequence. 


\subsection{Little 2-cubes and braid arrangements}

The space $\mathcal{C}_{\ell}(k)$ of $k$ little $\ell$-cubes is homotopy equivalent to the configuration space $F\left(\mathbb{R}^{\ell}, k\right)$ of $k$ distinct points in $\mathbb{R}^{\ell}$. When $\ell=2$,

$$
\mathcal{C}_{2}(k) \simeq F(\mathbb{C}, k)=\left\{\left(z_{1}, \ldots, z_{k}\right) \in \mathbb{C}^{k} \mid z_{i} \neq z_{j} \text { if } i \neq j\right\}
$$

For $1 \leq i<j \leq k$, define a hyperplane in $\mathbb{R}^{k}$,

$$
L_{i, j}=\left\{\left(x_{1}, \ldots, x_{k}\right) \in \mathbb{R}^{k} \mid x_{i}=x_{j}\right\}
$$

Then $F(\mathbb{C}, k)$ is the complement of the complexification of the real central hyperplane arrangement $\left\{L_{i, j} \mid 1 \leq i<j \leq k\right\}$,

$$
F(\mathbb{C}, k)=\mathbb{C}^{k}-\bigcup_{1 \leq i<j \leq k} L_{i, j} \otimes \mathbb{C} .
$$

Hyperplanes in this arrangement have the line $\left\{x_{1}=\cdots=x_{k}\right\}$ in common. To make it essential, let

$$
\mathfrak{h}_{k}=\left\{\left(x_{1}, \ldots, x_{k}\right) \in \mathbb{R}^{k} \mid x_{1}+\cdots+x_{k}=0\right\} \quad \text { and } \quad L_{i, j}^{\prime}=L_{i, j} \cap \mathfrak{h}_{k}
$$

The arrangement $\mathcal{A}_{k-1}=\left\{L_{i, j}^{\prime} \mid 1 \leq i<j \leq k\right\}$ is essential and we have homotopy equivalences

$$
\mathcal{C}_{2}(k) \simeq F(\mathbb{C}, k)=\mathbb{C}^{k}-\bigcup_{1 \leq i<j \leq k} L_{i, j} \otimes \mathbb{C} \simeq \mathfrak{h}_{k} \otimes \mathbb{C}-\bigcup_{1 \leq i<j \leq k} L_{i, j}^{\prime} \otimes \mathbb{C}
$$

Note that these homotopy equivalences respect the right action of the symmetric group $\Sigma_{k}$.

The homotopy types of complements of complexified arrangements have been actively studied by many people. One of the most interesting and useful constructions in this area is a result of M. Salvetti [Sal87], who constructed a finite simplicial complex $\operatorname{Sal}(\mathcal{A})$ for a real central essential arrangement $\mathcal{A}$, embedded in the complement of the complexification of $\mathcal{A}$ as a deformation retract. Thus we obtain

$$
\mathcal{C}_{2}(k) \simeq \mathfrak{h}_{k} \otimes \mathbb{C}-\bigcup_{1 \leq i<j \leq k} L_{i, j}^{\prime} \otimes \mathbb{C} \simeq \operatorname{Sal}\left(\mathcal{A}_{k-1}\right)
$$

There should be a combinatorial meaning of the filtration $(5)$ on $\mathcal{C}_{2}(k)$ when translated to the Salvetti complex $\operatorname{Sal}\left(\mathcal{A}_{k-1}\right)$ of the braid arrangement under the homotopy equivalence (6). 


\subsection{Statement of results}

For any real central hyperplane arrangement $\mathcal{A}$, Salvetti, on the other hand, defined a structure of regular cell complex on $\operatorname{Sal}(\mathcal{A})$ by combining simplices by using the combinatorial structure of the face lattice of $\mathcal{A}$.

It turns out that the filtration (5) on $\mathcal{C}_{2}(k)$ designed for the Eilenberg-Moore spectral sequence (2) coincides with the filtration defined by the cellular structure on $\operatorname{Sal}\left(\mathcal{A}_{k-1}\right)$ under the homotopy equivalence (6).

Theorem (Theorem 4.12). Let $F_{-s} \operatorname{Sal}\left(\mathcal{A}_{k-1}\right)$ be the $(k-s)$-skeleton of $\operatorname{Sal}\left(\mathcal{A}_{k-1}\right)$ under the cellular structure defined in [Sal87]. Then the homotopy equivalence

$$
\varphi_{k}: \operatorname{Sal}\left(\mathcal{A}_{k-1}\right) \stackrel{\simeq}{\rightarrow} \mathcal{C}_{2}(k)
$$

in (6) preserves filtrations and induces a $\Sigma_{k}$-equivariant homotopy equivalence on each subquotient,

$$
\varphi_{k}: F_{-s} \operatorname{Sal}\left(\mathcal{A}_{k-1}\right) / F_{-s-1} \operatorname{Sal}\left(\mathcal{A}_{k-1}\right) \stackrel{\sim}{\rightarrow} F_{-s} \mathcal{C}_{2}(k) / F_{-s-1} \mathcal{C}_{2}(k) .
$$

A filtration on each $\mathcal{C}_{2}(k)$ induces a spectral sequence converging to the homology $h_{*}\left(\mathcal{C}_{2}(k)_{+} \wedge_{\Sigma_{k}} X^{\wedge k}\right)$ and the Eilenberg-Moore spectral sequence (2) decomposes into a direct sum of these small spectral sequences. Let $\left\{E^{r}\left(\mathcal{A}_{k-1}\right)\right\}$ be the spectral sequence for $h_{*}\left(\operatorname{Sal}\left(\mathcal{A}_{k-1}\right)_{+} \wedge_{\Sigma_{k}} X^{\wedge k}\right)$ defined by the skeletal filtration on $\operatorname{Sal}\left(\mathcal{A}_{k-1}\right)$. Then the $E^{1}$-term of the spectral sequence defined in [Tam94] for $h_{*}\left(\Omega^{2} \Sigma^{2} X\right)$ can be identified as follows:

$$
\begin{aligned}
E_{-s, t}^{1} & =\bigoplus_{k \geq 0} E_{-s, t}^{1}\left(\mathcal{A}_{k-1}\right) \cong \bigoplus_{k \geq 0} C_{k-s}\left(\operatorname{Sal}\left(\mathcal{A}_{k-1}\right)\right) \otimes_{\Sigma_{k}} \tilde{h}_{t-k}\left(X^{\wedge k}\right) \\
& \cong \bigoplus_{k \geq 0} C_{k-s}\left(\operatorname{Sal}\left(\mathcal{A}_{k-1}\right)\right) \otimes_{\Sigma_{k}} \tilde{h}_{t}\left((\Sigma X)^{\wedge k}\right)
\end{aligned}
$$

where $C_{*}(-)$ denotes the cellular chain complex functor. This is an isomorphism of chain complexes. When $h_{*}(-)$ is multiplicative and satisfies the strong form of the Künneth formula, we have

$$
E_{-s, *}^{1} \cong \bigoplus_{k \geq 0} C_{k-s}\left(\operatorname{Sal}\left(\mathcal{A}_{k-1}\right)\right) \otimes_{\Sigma_{k}} \tilde{h}_{*}(\Sigma X)^{\otimes k} .
$$

The $(k-s)$-cells in $\operatorname{Sal}\left(\mathcal{A}_{k-1}\right)$ are labeled by pairs of an ordered partition $\lambda$ and a permutation $\sigma$ which is a subdivision of $\lambda$, in which case we write $\lambda \leq \sigma$. Then we have

$$
\begin{aligned}
E_{-s, *}^{1} & \cong \bigoplus_{k \geq 0} \mathbb{Z}\left\langle[D(\lambda, \sigma)] \mid \lambda \in \Pi_{k, k-s}, \sigma \in \Sigma_{k}, \lambda \leq \sigma\right\rangle \otimes_{\Sigma_{k}} \tilde{h}_{*}(\Sigma X)^{\otimes k} \\
& \cong \bigoplus_{k \geq 0} \mathbb{Z}\left\langle[D(\lambda,(1|\cdots| k))] \mid \lambda \in O_{k, k-s}\right\rangle \otimes \tilde{h}_{*}(\Sigma X)^{\otimes k},
\end{aligned}
$$

where $(1|\cdots| k) \in \Sigma_{k}$ is the identity and $\Pi_{k, k-s}$ and $O_{k, k-s}$ are the set of ordered partitions and of order-preserving partitions of rank $k-s$, respectively. See $\S 4$ for precise definitions. 
On the other hand, it is proved in [Tam07] that the $E^{1}$-term of the spectral sequence is isomorphic to the cobar complex of $h_{*}\left(\Omega \Sigma^{2} X\right) \cong T\left(\tilde{h}_{*}(\Sigma X)\right)$, the tensor algebra on $\tilde{h}_{*}(\Sigma X)$. This means that the cellular structure of the Salvetti complex describes the iterated cobar construction. This immediately gives us the description of the cobar differential stated in Introduction of [Tam07]. (For the precise definitions, see p. 823 and p. 825.)

Theorem (Corollary 4.18). The first differential $d^{1}$ on the spectral sequence is given by the following formula:

$$
\begin{aligned}
d_{-s, *}^{1}\left([D(\lambda,(1|\cdots| k))] \otimes\left[x_{1}|\cdots| x_{k}\right]\right) & \\
\quad= & \sum_{\tau \in O_{k, k-s-1}, \lambda<\tau}[D(\tau,(1|\cdots| k))] \otimes\left(\sum_{\rho \in S_{t(\tau)}} \operatorname{sgn}(\rho)\left[x_{1}|\cdots| x_{k}\right] \cdot \rho\right),
\end{aligned}
$$

where $S_{t(\tau)}$ is the set of shuffles of the same type as $\tau$.

With this description, it is easy to see that the first differentials are induced by space-level shuffles

$$
\bigvee_{\lambda \in O_{k, k-s}} S_{(\lambda,(1|\cdots| k))}^{k-s} \wedge(\Sigma X)^{\wedge k} \rightarrow \Sigma\left(\bigvee_{\tau \in O_{k, k-s-1, \lambda<\tau}} S_{(\tau,(1|\cdots| k))}^{k-s-1} \wedge(\Sigma X)^{\wedge k}\right)
$$

where $S_{(\lambda,(1|\cdots| k))}^{k-s}$ and $S_{(\tau,(1|\cdots| k)}^{k-s-1}$ are copies of the spheres $S^{k-s}$ and $S^{k-s-1}$, respectively.

This map may be of some use to study maps between wedge powers of suspended spaces.

The spectral sequence (2) is defined not only for $\ell=2$ but for all $\ell \geq 1$. On the other hand, it was observed by Björner and Ziegler [BZ92] and by De Concini and Salvetti [DCS00] that the construction of $\operatorname{Sal}(\mathcal{A})$ can be extended to subspace arrangements of higher codimensions. For a real central essential arrangement $\mathcal{A}$ in a vector space $V$, there is a simplicial complex $\operatorname{Sal}^{(\ell-1)}(\mathcal{A})$ embedded in the complement of the $\ell$-dimensionalization,

$$
\mathrm{Sal}^{(\ell-1)}(\mathcal{A}) \hookrightarrow V \otimes \mathbb{R}^{\ell}-\bigcup_{L \in \mathcal{A}} L \otimes \mathbb{R}^{\ell},
$$

as a deformation retract. We can generalize the cellular structure of $\operatorname{Sal}(\mathcal{A})=\operatorname{Sal}^{(1)}(\mathcal{A})$ to $\mathrm{Sal}^{(\ell-1)}(\mathcal{A})$ to make it a regular cell complex. Under the stable homotopy equivalence

$$
\Omega^{\ell} \Sigma^{\ell} X \simeq \bigvee_{k} \mathcal{C}_{\ell}(k)_{+} \wedge X^{\wedge k} \simeq \bigvee_{k} F\left(\mathbb{R}^{\ell}, k\right)_{+} \wedge X^{\wedge k} \simeq \bigvee_{k} \operatorname{Sal}^{(\ell-1)}\left(\mathcal{A}_{k-1}\right)_{+} \wedge X^{\wedge k},
$$

the skeletal filtration on $\mathrm{Sal}^{(\ell-1)}\left(\mathcal{A}_{k-1}\right)$ induces a spectral sequence that computes $h_{*}\left(\Omega^{\ell} \Sigma^{\ell} X\right)$.

Theorem (Theorem 5.35). For any homology theory there exists a spectral sequence

$$
E^{1} \cong \bigoplus_{k} C_{*}\left(\operatorname{Sal}^{(\ell-1)}\left(\mathcal{A}_{k-1}\right)\right) \otimes_{\Sigma_{k}} \tilde{h}_{*}\left(X^{\wedge k}\right) \Rightarrow h_{*}\left(\Omega^{\ell} \Sigma^{\ell} X\right),
$$

which is a direct sum of spectral sequences each of which strongly converges to the corresponding direct summand in (3). 
The spectral sequence is different from the Eilenberg-Moore spectral sequence (2) when $\ell>2$. It is also different from the spectral sequence studied by Ahearn and Kuhn [AK02]. Thus we obtain a new spectral sequence which can be used to compute $h_{*}\left(\Omega^{\ell} \Sigma^{\ell} X\right)$. As we will see in $\S 6$, our spectral sequence is much finer than Ahearn-Kuhn's.

It was pointed out by the referee that the construction of the higher order Salvetti complex $\mathrm{Sal}^{(\ell-1)}\left(\mathcal{A}_{k}\right)$ for the braid arrangement together with the skeletal filtration could be obtained by using Milgram's model [Mil66] for $\Omega^{\ell} \Sigma^{\ell} X$. We accomplish this by using a categorical variant of Milgram's construction found by Balteanu, Fiedorowicz, Schwänzl, and Vogt [BFSV03] in $§ 6$.

The organization of this paper is as follows. We recall the construction of the Salvetti complex in $\$ 2$. Salvetti's construction has been intensively studied and several alternative descriptions and interpretations are known. We use the description in terms of matroid product developed in [GR89], [Arv91], and [BZ92]. The properties of the filtration on the space of little cubes are summarized in $\S 3$. After these preliminary sections, we compare the Salvetti complex for the braid arrangement $\mathcal{A}_{k-1}$ and the filtration on the space of $k$ little 2-cubes in $\S 4$ and prove Theorem 4.12. Higher order analogues are discussed in $\S 5$, where we construct the spectral sequence (7) by using oriented matroids. Comparisons with other spectral sequences that compute the homology of iterated loop spaces and with Milgram's construction are given in $§ 6$.

\section{The Salvetti complex}

For a real central arrangement $\mathcal{A}$ in $\mathbb{R}^{n}$, Salvetti defined a simplicial complex $\operatorname{Sal}(\mathcal{A})$ embedded in the complement of the complexification of the arrangement in $\mathbb{C}^{n}$. Let us recall the construction of $\operatorname{Sal}(\mathcal{A})$. Although the combinatorial information on a real central arrangement can be translated into the language of oriented matroids and the construction of the Salvetti complex can be generalized to oriented matroids, we follow the original treatment [Sal87] in this section, since we need explicit descriptions of vertices for later use. We use the matroid description of the Salvetti complex in $§ 5$.

Let us first fix the notation and terminology. Let $\mathcal{A}=\left\{M_{j}\right\}_{j \in J}$ be a central arrangement of hyperplanes in $\mathbb{R}^{n}$. The arrangement $\mathcal{A}$ defines a stratification of $\mathbb{R}^{n}$ :

$$
\begin{aligned}
M^{0} & =\mathbb{R}^{n}-\bigcup_{j \in J} M_{j}, \\
M^{1} & =\bigcup_{j \in J}\left(M_{j}-\bigcup_{k \neq j} M_{j} \cap M_{k}\right), \\
& \vdots \\
M^{|J|} & =\bigcap_{j \in J} M_{j}, \\
\mathbb{R}^{n} & =M^{0} \cup M^{1} \cup \cdots \cup M^{|J|} .
\end{aligned}
$$

The connected components of each stratum are called faces and a face in the top stratum is called a chamber. The sets of all faces and of faces of codimension $i$ are denoted 
by $\mathcal{F}(\mathcal{A})$ and $\mathcal{F}^{i}(\mathcal{A})$. In particular, $\mathcal{F}^{0}(\mathcal{A})$ is the set of chambers. We need an ordering in $\mathcal{F}(\mathcal{A})$. Although a popular way to define an ordering is by "reverse inclusion", Salvetti defines an ordering as follows:

$$
F \geq F^{\prime} \Leftrightarrow \bar{F} \supset F^{\prime} .
$$

In order to define a simplicial complex, we need vertices. For each face $F$, we choose a point $w(F) \in F$ and the collections of these points are denoted by

$$
\mathcal{V}(\mathcal{A})=\{w(F) \mid F \in \mathcal{F}(\mathcal{A})\}, \quad \mathcal{V}^{i}(\mathcal{A})=\left\{w(F) \mid F \in \mathcal{F}^{i}(\mathcal{A})\right\} .
$$

These are points in $\mathbb{R}^{n}$. We need to add some imaginary coordinates to obtain points in $\mathbb{C}^{n}$. Salvetti defines the imaginary part by using the following fact.

Lemma 2.1. For $v \in \mathcal{V}^{0}(\mathcal{A})$ and $F \in \mathcal{F}(\mathcal{A})$, there exists a unique point $w(v, F) \in$ $\mathcal{V}^{0}(\mathcal{A})$ with the following properties:

1. $v$ and $w(v, F)$ belong to the same chamber of $\mathcal{A}_{\supset F}$, which is the arrangement defined by

$$
\mathcal{A}_{\supset F}=\{H \in \mathcal{A} \mid H \supset F\} .
$$

2. $w(v, F)$ belongs to a chamber in $\left\{C \in \mathcal{F}^{0}(\mathcal{A}) \mid C \geq F\right\}$.

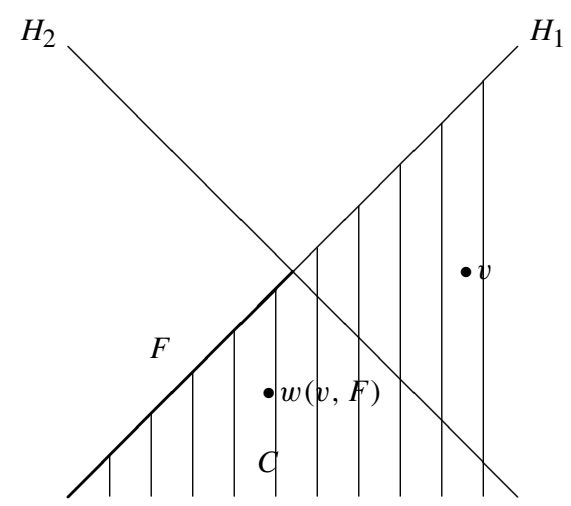

Fig. 1. $w(v, F)$

The vertices of the Salvetti complex are given by

$$
w(F)+i(w(v, F)-w(F))
$$

for each element $v \in \mathcal{V}^{0}(\mathcal{A})$ and a face $F$. Note that $w(v, F)$ is a vertex corresponding to a chamber. Thus the vertices of the Salvetti complex are given by

$$
v(F, C)=w(F)+i(w(C)-w(F))
$$

for a face $F$ and a chamber $C$ satisfying a certain condition. When $v$ varies, all points in the chamber $C$ with $C \geq F$ appear as $w(v, F)$. Thus we have the following.

Definition 2.2. For a real central arrangement $\mathcal{A}$, define the vertex set by

$$
\operatorname{sk}_{0}(\operatorname{Sal}(\mathcal{A}))=\left\{v(F, C) \mid F \in \mathcal{F}(\mathcal{A}), C \in \mathcal{F}^{0}(\mathcal{A}), F \leq C\right\}
$$


In order to define higher dimensional simplices, we need to specify a condition for a collection of vertices to form a simplex. The condition can be described by using a "face-chamber" pairing

$$
\mathcal{F}(\mathcal{A}) \times \mathcal{F}^{0}(\mathcal{A}) \rightarrow \mathcal{F}^{0}(\mathcal{A})
$$

This can be extended to a "face-face" pairing

$$
\mathcal{F}(\mathcal{A}) \times \mathcal{F}(\mathcal{A}) \rightarrow \mathcal{F}(\mathcal{A})
$$

Proposition 2.3. For $F, G \in \mathcal{F}(\mathcal{A})$, there exists a unique face $F \circ G \in \mathcal{F}(\mathcal{A})$ with the following properties. Let $H \in \mathcal{F}\left(\mathcal{A}_{\supset|F|}\right)$ be the unique face with $G \subset H$. Then $F \circ G$ is the unique face with $F \circ G \subset H$ and $F \leq F \circ G$.

Lemma 2.4 ([Arv91]). The above face-face pairing has the following properties:

1. The pairing is associative.

2. If $G$ is a chamber, then so is $F \circ G$.

3. If $F$ is a chamber, then $F \circ G=F$.

4. If $G \leq G^{\prime}$, then $F \circ G \leq F \circ G^{\prime}$.

With this pairing, the simplices of the Salvetti complex can be defined as follows.

Definition 2.5. For a chain $F_{0}>F_{1}>\cdots>F_{j}$ in $\mathcal{F}(\mathcal{A})$ and a chamber $C$ with $C \geq F_{j}$, define a simplex by

$$
s\left(F_{0}, \ldots, F_{j} ; C\right)=\operatorname{Conv}\left(\left\{v\left(F_{0}, F_{0} \circ C\right), \ldots, v\left(F_{j}, F_{j} \circ C\right)\right\}\right)
$$

where $\operatorname{Conv}(S)$ denotes the convex hull of $S$.

The Salvetti complex is defined by

$$
\operatorname{Sal}(\mathcal{A})=\bigcup_{j}\left\{s\left(F_{0}, \ldots, F_{j} ; C\right) \mid F_{0}>\cdots>F_{j}, C \geq F_{j}, C \in \mathcal{F}^{0}(\mathcal{A})\right\} .
$$

Salvetti proves that $\operatorname{Sal}(\mathcal{A})$ is a deformation retract of the complement of the complexification $\mathcal{A}^{\mathbb{C}}$ of $\mathcal{A}$.

Theorem 2.6 (Salvetti [Sal87]). $\operatorname{Sal}(\mathcal{A})$ is contained in the complement of the complexification of $\mathcal{A}$ and the inclusion is a homotopy equivalence,

$$
\operatorname{Sal}(\mathcal{A}) \simeq \mathbb{C}^{n}-\bigcup_{M \in \mathcal{A}} M^{\mathbb{C}}
$$

where $M^{\mathbb{C}}$ is the complexification of $M$.

Although $\operatorname{Sal}(\mathcal{A})$ has a nice simplicial structure, it is more convenient to combine simplices which form a cell. In fact, Salvetti defines a CW-structure. Using the face-face pairing, we have the following description. 
Definition 2.7. For $F \in \mathcal{F}(\mathcal{A})$ and $C \in \mathcal{F}^{0}(\mathcal{A})$ with $F \leq C$, define a subset of $\operatorname{sk}_{0}(\operatorname{Sal}(\mathcal{A}))$ by

$$
\mathcal{D}(F, C)=\{v(G, G \circ C) \mid G \geq F\} .
$$

This set is regarded as a poset via

$$
v(G, G \circ C) \leq v(H, H \circ C) \Leftrightarrow G \leq H .
$$

The (geometric realization of the) order complex of $\mathcal{D}(F, C)$ is denoted by $D(F, C)$.

Salvetti proves the following (see [Arv91]).

Lemma 2.8. The complex $D(F, C)$ has the following properties:

1. The inclusion of vertices induces a simplicial embedding

$$
D(F, C) \hookrightarrow \operatorname{Sal}(\mathcal{A}) .
$$

2. $D(F, C)$ is homeomorphic to a disk of dimension codim $F$.

3. The boundary of $D(F, C)$ is given by

$$
\partial D(F, C)=\bigcup_{G>F} D(G, G \circ C) .
$$

4. The decomposition

$$
\operatorname{Sal}(\mathcal{A})=\bigcup_{v(F, C) \in \mathrm{sk}_{0}(\operatorname{Sal}(\mathcal{A}))}(D(F, C)-\partial D(F, C))
$$

gives a structure of a finite regular cell complex.

\section{The gravity filtration on little cubes}

In [Tam94], the author introduced a filtration on the space of $k$ little $\ell$-cubes, $\mathcal{C}_{\ell}(k)$, for all $\ell$ and $k$. The subquotients $F_{-s} \mathcal{C}_{\ell}(k) / F_{-s-1} \mathcal{C}_{\ell}(k)$ are analyzed in [Tam07]. In this section, we recall the definition and basic properties of this filtration.

$\mathcal{C}_{\ell}(k)$ is the space of little cubes in $[-1,1]^{\ell}$. In particular, we regard an element of $\mathcal{C}_{\ell}(1)$ as an embedding

$$
c:(-1,1)^{\ell} \rightarrow(-1,1)^{\ell} .
$$

In order to compare it with the Salvetti complex in the next section, however, it is more convenient to consider cubes in $\mathbb{R}^{\ell}$.

Convention 3.1. In the rest of this paper, $\mathcal{C}_{\ell}(k)$ denotes the space of $k$ little $\ell$-cubes in $\mathbb{R}^{\ell}$ whose images have pairwise disjoint interiors and whose edges are parallel to coordinate axes. However, when we draw a picture, we use the usual picture for little cubes, i.e. "nonoverlapping small boxes in a big box".

We also use a description of little cubes with centers and radii. For example, a little 1 -cube

$$
b:(-1,1) \hookrightarrow \mathbb{R}
$$

can be given by $b(t)=R t+C$ for $R>0$ and $C \in \mathbb{R}$. 
Let us begin by recalling the definition of the filtration $\left\{F_{-s} \mathcal{C}_{\ell}(k)\right\}_{s}$ on $\mathcal{C}_{\ell}(k)$ from [Tam94]. The definition proceeds by defining a continuous function

$$
u_{s}: F_{-s} \mathcal{C}_{\ell}(k) \rightarrow[0,1]
$$

which measures the overlapping of the first coordinates of cubes inductively and by putting

$$
F_{-s-1} \mathcal{C}=u_{s}^{-1}(0)
$$

The author used little cubes in $[-1,1]^{\ell}$ in [Tam94], but the definition of the functions $u_{s}$ works without a change. The construction of the function $u_{s}$ is given as follows.

Definition 3.2. For an element $b \in \mathcal{C}_{1}(1)$ given by $b(t)=R t+C$ and $x \in \mathbb{R}$, define

$$
d(x, b)=\frac{2 R-|| C+R-x|-| C-R-x||}{2 R} .
$$

For two little 1-cubes $b, b^{\prime} \in \mathcal{C}_{1}(1)$, define

$$
\operatorname{dis}\left(b, b^{\prime}\right)=\min \left\{d\left(b(0), b^{\prime}\right), d\left(b^{\prime}(0), b\right)\right\} .
$$

Then we have a continuous map

$$
\text { dis : } \mathcal{C}_{1}(1) \times \mathcal{C}_{1}(1) \rightarrow[0,1] .
$$

Composing it with the projection

$$
\operatorname{pr}_{1}: \mathcal{C}_{\ell}(1) \rightarrow \mathcal{C}_{1}(1)
$$

onto the first coordinate, we obtain a continuous map

$$
\text { dis : } \mathcal{C}_{\ell}(1) \times \mathcal{C}_{\ell}(1) \rightarrow[0,1] .
$$

These maps are designed to have the following property.

Lemma 3.3. For $b \in \mathcal{C}_{1}(1), d(x, b)=0$ if and only if $x \notin \operatorname{Im} b$, and $d(x, b)=1$ if and only if $x=b(0)$. Thus, for $b, b^{\prime} \in \mathcal{C}_{1}(1), \operatorname{dis}\left(b, b^{\prime}\right)=0$ if and only if the center of $b$ belongs to the image of $b^{\prime}$ and vice versa. And $\operatorname{dis}\left(b, b^{\prime}\right)=1$ if and only if the centers of $b$ and $b^{\prime}$ coincide.

We define a function which measures the overlapping of little cubes with respect to a partition of $\{1, \ldots, k\}$. Here a partition always means an ordered partition.

Definition 3.4. A partition of $\{1, \ldots, k\}$ is a surjective map

$$
\lambda:\{1, \ldots, k\} \rightarrow\{1, \ldots, k-r\}
$$

for some $0 \leq r<k$. The number $r$ is called the rank of this partition.

The set of partitions of $\{1, \ldots, k\}$ is denoted by $\Pi_{k}$. The subset of rank $r$ partitions is denoted by $\Pi_{k, r}$. 
Note that rank 0 partitions are nothing but elements of $\Sigma_{k}$.

Definition 3.5. For $c=\left(c_{1}, \ldots, c_{k}\right) \in \mathcal{C}_{\ell}(k)$ and a nonempty subset $S \subset\{1, \ldots, k\}$, define

$$
O L(c, S)=\min \left\{\operatorname{dis}\left(c_{i}, c_{j}\right) \mid i, j \in S\right\} .
$$

For a partition $\lambda:\{1, \ldots, k\} \rightarrow\{1, \ldots, s\}$ of $\{1, \ldots, k\}$ of rank $k-s$, define

$$
\operatorname{MOL}(c, \lambda)=\min \left\{O L\left(c, \lambda^{-1}(i)\right) \mid 1 \leq i \leq s\right\} .
$$

Note that we have continuous functions

$$
O L(-, S): \mathcal{C}_{\ell}(k) \rightarrow[0,1], \quad \operatorname{MOL}(-, \lambda): \mathcal{C}_{\ell}(k) \rightarrow[0,1]
$$

With these functions, we define the gravity filtration on $\mathcal{C}_{\ell}(k)$ as follows.

Definition 3.6. Define

$$
F_{0} \mathcal{C}_{\ell}(k)=F_{-1} \mathcal{C}_{\ell}(k)=\mathcal{C}_{\ell}(k)
$$

Suppose we have defined $F_{-s} \mathcal{C}_{\ell}(k)$. Define

$$
u_{s}: F_{-s} \mathcal{C}_{\ell}(k) \rightarrow[0,1] \quad \text { by } \quad u_{s}(\boldsymbol{c})=\max \left\{\operatorname{MOL}(\boldsymbol{c}, \lambda) \mid \lambda \in \Pi_{k, k-s}\right\} .
$$

Now define

$$
F_{-s-1} \mathcal{C}_{\ell}(k)=u_{s}^{-1}(0)
$$

We have a filtration

$$
\emptyset=F_{-k-1} \mathcal{C}_{\ell}(k) \subset F_{-k} \mathcal{C}_{\ell}(k) \subset \cdots \subset F_{-2} \mathcal{C}_{\ell}(k) \subset F_{-1} \mathcal{C}_{\ell}(k)=F_{0} \mathcal{C}_{\ell}(k)=\mathcal{C}_{\ell}(k) .
$$

It was proved in [Tam94] that each inclusion is an NDR pair.

In order to state the result in [Tam07], we need the following notation.

Definition 3.7. Fix $\varepsilon>0$, and define a $(k-1)$-dimensional convex polytope $P^{k}$ in the hyperplane $\mathfrak{h}_{k}=\left\{\left(t_{1}, \ldots, t_{k}\right) \in \mathbb{R}^{k} \mid t_{1}+\cdots+t_{k}=0\right\}$ by

$$
P^{k}=\left\{\left(t_{1}, \ldots, t_{k}\right) \in \mathfrak{h}_{k}|| t_{i}-t_{j} \mid<\varepsilon \text { for } i \neq j\right\} .
$$

For a subset $S=\left\{s_{1}, \ldots, s_{\ell}\right\} \subset\{1, \ldots, k\}$, define

$$
\begin{aligned}
\mathbb{R}^{S} & =\operatorname{Map}(S, \mathbb{R})=\left\{\left(t_{s_{1}}, \ldots, t_{s_{\ell}}\right) \mid t_{s_{1}}, \ldots, t_{s_{\ell}} \in \mathbb{R}\right\}, \\
\mathfrak{h}_{S} & =\left\{\left(t_{s_{1}}, \ldots, t_{s_{\ell}}\right) \in \mathbb{R}^{S} \mid t_{s_{1}}+\cdots+t_{s_{\ell}}=0\right\}, \\
P^{S} & =\left\{\left(t_{s_{1}}, \ldots, t_{s_{\ell}}\right) \in \mathfrak{h}_{S}|| t_{s}-t_{s^{\prime}} \mid<\varepsilon \text { for } s \neq s^{\prime}\right\} .
\end{aligned}
$$

Also for little cubes, we denote

$$
\mathcal{C}_{\ell}(S)=\left\{\left(c_{s_{1}}, \ldots, c_{s_{\ell}}\right) \in \operatorname{Map}\left(S, \mathcal{C}_{\ell}(1)\right) \mid \operatorname{Im} c_{s_{i}} \cap \operatorname{Im} c_{s_{j}}=\emptyset\right\} .
$$

With this notation, it is proved in [Tam07] that 
Proposition 3.8. We have a $\Sigma_{k}$-equivariant homotopy equivalence

$$
\begin{aligned}
& F_{-s} \mathcal{C}_{\ell}(k) / F_{-s-1} \mathcal{C}_{\ell}(k) \simeq \\
& \bigvee_{\lambda \in \Pi_{k, k-s}} \mathcal{C}_{\ell-1}\left(\lambda^{-1}(1)\right)_{+} \wedge\left(P^{\lambda^{-1}(1)} / \partial P^{\lambda^{-1}(1)}\right) \wedge \cdots \wedge \mathcal{C}_{\ell-1}\left(\lambda^{-1}(s)\right)_{+} \wedge\left(P^{\lambda^{-1}(s)} / \partial P^{\lambda^{-1}(s)}\right) \\
& \quad=\bigvee_{\lambda \in \Pi_{k, k-s}} \mathcal{C}_{\ell-1}(\lambda)_{+} \wedge P^{\lambda} / \partial P^{\lambda}
\end{aligned}
$$

where

$$
\begin{aligned}
\mathcal{C}_{\ell-1}(\lambda) & =\mathcal{C}_{\ell-1}\left(\lambda^{-1}(1)\right) \times \cdots \times \mathcal{C}_{\ell-1}\left(\lambda^{-1}(s)\right), \\
P^{\lambda} & =P^{\lambda^{-1}(1)} \times \cdots \times P^{\lambda^{-1}(s)}
\end{aligned}
$$

for a partition $\lambda$ of rank $k-s$.

This homotopy equivalence is proved as follows. The first step is to replace $F_{-s} \mathcal{C}_{\ell}(k)$ by horizontally decomposable cubes.

Definition 3.9. Let $\mathcal{D}_{\ell}^{s}(k)$ be the subset of $\mathcal{C}_{\ell}(k)$ consisting of cubes which are horizontally decomposable into $s$ collections.

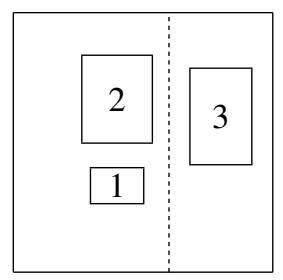

A more precise definition can be given in terms of the operad structure map. See [Tam07] for details.

Note that $\mathcal{D}_{\ell}^{s}(k)$ is included in $F_{-s} \mathcal{C}_{\ell}(k)$.

Lemma 3.10. Inclusion induces a $\Sigma_{k}$-equivariant homotopy equivalence

$$
\mathcal{D}_{\ell}^{s}(k) / \mathcal{D}_{\ell}^{s+1}(k) \simeq F_{-s} \mathcal{C}_{\ell}(k) / F_{-s-1} \mathcal{C}_{\ell}(k) .
$$

We also have

$\mathcal{D}_{\ell}^{s}(k) / \mathcal{D}_{\ell}^{s+1}(k) \simeq \bigvee_{\lambda \in \Pi_{k, s}} \mathcal{D}_{\ell}^{1}\left(\lambda^{-1}(1)\right) / \mathcal{D}_{\ell}^{2}\left(\lambda^{-1}(1)\right) \wedge \cdots \wedge \mathcal{D}_{\ell}^{1}\left(\lambda^{-1}(s)\right) / \mathcal{D}_{\ell}^{2}\left(\lambda^{-1}(s)\right)$

Thus it is enough to analyze $\mathcal{D}_{\ell}^{1}(k) / \mathcal{D}_{\ell}^{2}(k)$. We can make $\mathcal{D}_{\ell}^{1}(k)$ even smaller by removing unnecessary cubes. Note that the following element in $\mathcal{D}^{1}(3)$ can be moved into $\mathcal{D}^{2}(3)$ by shrinking the first coordinates of cubes. 


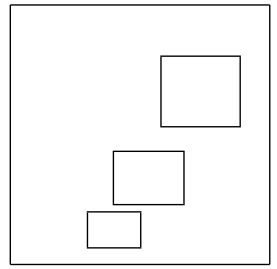

All we need is cubes in $\mathcal{D}_{\ell}^{1}(k)$ which can be skewered vertically.

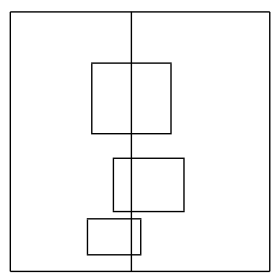

Definition 3.11. Define $G_{-s} \mathcal{C}_{\ell}(k)$ to be the subset of $\mathcal{D}_{\ell}^{s}(k)$ consisting of cubes $\left(c_{1}, \ldots, c_{j}\right)$ which cannot be decomposed into $s-1$ collections of cubes each of which can be skewered by a vertical line (hyperplane) intersecting each interior.

Lemma 3.12. Inclusion induces a $\Sigma_{k}$-equivariant homotopy equivalence

$$
G_{-1} \mathcal{C}_{\ell}(k) / G_{-2} \mathcal{C}_{\ell}(k) \simeq \mathcal{D}_{\ell}^{1}(k) / \mathcal{D}_{\ell}^{2}(k)
$$

Finally we adjust the radii of the first coordinates of cubes in $G_{-1} \mathcal{C}_{\ell}(k)$ so that they have the same fixed radii.

Definition 3.13. For $\varepsilon>0$, let $\mathcal{C}_{\ell}^{\varepsilon}(k)$ be the subspace of $\mathcal{C}_{\ell}(k)$ consisting of cubes having the radii of the first coordinates equal to $\varepsilon$.

Lemma 3.14. Inclusion induces a $\Sigma_{k}$-equivariant homotopy equivalence

$$
G_{-1} \mathcal{C}_{\ell}(k) \cap \mathcal{C}_{\ell}^{\varepsilon}(k) / G_{-2} \mathcal{C}_{\ell}(k) \cap \mathcal{C}_{\ell}^{\varepsilon}(k) \simeq G_{-1} \mathcal{C}_{\ell}(k) / G_{-2} \mathcal{C}_{\ell}(k) .
$$

Now it is easy to describe $G_{-1} \mathcal{C}_{\ell}(k) \cap \mathcal{C}_{\ell}^{\varepsilon}(k) / G_{-2} \mathcal{C}_{\ell}(k) \cap \mathcal{C}_{\ell}^{\varepsilon}(k)$.

Lemma 3.15. Define

$$
\begin{aligned}
\widetilde{P}^{k} & =\left\{\left(t_{1}, \ldots, t_{k}\right) \in \mathbb{R}^{k}|| t_{i}-t_{j} \mid<\varepsilon \text { for all } i, j\right\}, \\
d \widetilde{P}^{k} & =\left\{\left(t_{1}, \ldots, t_{k}\right) \in \mathbb{R}^{k}|| t_{i}-t_{j} \mid=\varepsilon \text { for some } i \neq j\right\} .
\end{aligned}
$$

Then we have a $\Sigma_{k}$-equivariant homeomorphism

$$
G_{-1} \mathcal{C}_{\ell}(k) \cap \mathcal{C}_{\ell}^{\varepsilon}(k) / G_{-2} \mathcal{C}_{\ell}(k) \cap \mathcal{C}_{\ell}^{\varepsilon}(k) \cong \widetilde{P}^{k} / d \widetilde{P}^{k} \wedge \mathcal{C}_{\ell-1}(k)_{+} .
$$


Under the decomposition of the permutation representation

$$
\mathbb{R}^{k} \cong \mathfrak{h}_{k} \oplus\{(t, \ldots, t) \mid t \in \mathbb{R}\},
$$

we see that inclusion induces a $\Sigma_{k}$-equivariant homotopy equivalence

$$
P^{k} / \partial P^{k} \simeq \widetilde{P}^{k} / d \widetilde{P}^{k} .
$$

The homotopy equivalence in Proposition 3.8 is induced by the composition of the inclusions

$P^{\lambda^{-1}(i)} \hookrightarrow \widetilde{P}^{\lambda^{-1}(i)} \hookrightarrow G_{-1} \mathcal{C}_{\ell}\left(\lambda^{-1}(i)\right) \cap \mathcal{C}_{\ell}^{\varepsilon}\left(\lambda^{-1}(i)\right) \hookrightarrow G_{-1} \mathcal{C}_{\ell}\left(\lambda^{-1}(i)\right) \hookrightarrow \mathcal{D}_{\ell}^{1}\left(\lambda^{-1}(i)\right)$

together with the decomposition (8) and the homotopy equivalence in Lemma 3.10.

\section{Salvetti complexes for braid arrangements}

Consider the root system $A_{k-1}$, which is a collection of vectors in the Cartan subalgebra $\mathfrak{h}_{k}$ of $\mathfrak{s l}_{k}(\mathbb{R})$. We may regard

$$
\mathfrak{h}_{k}=\left\{\left(t_{1}, \ldots, t_{k}\right) \in \mathbb{R}^{k} \mid t_{1}+\cdots+t_{k}=0\right\}
$$

and the action of the Weyl group, which is the symmetric group $\Sigma_{k}$ on $k$ letters, on $\mathfrak{h}_{k}$ is generated by the reflections with respect to the hyperplanes

$$
L_{i, j}=\left\{\boldsymbol{x}=\left(x_{1}, \ldots, x_{k}\right) \in \mathbb{R}^{k} \mid x_{i}=x_{j}\right\} .
$$

The complement of the complexification of this arrangement

$$
\mathbb{C}^{k}-\bigcup_{i \neq j} L_{i, j} \otimes \mathbb{C}
$$

is nothing but the configuration space of $k$ points in $\mathbb{C}, F(\mathbb{C}, k)$, and, under the identification (9), it is $\Sigma_{k}$-equivariantly homotopy equivalent to the complement

$$
\mathfrak{h}_{k} \otimes \mathbb{C}-\bigcup_{i \neq j}\left(L_{i, j} \cap \mathfrak{h}_{k}\right) \otimes \mathbb{C} .
$$

In fact, the projections

$$
p: \mathbb{R}^{k} \rightarrow \mathfrak{h}_{k}, \quad p \otimes \mathbb{C}: \mathbb{C}^{k} \rightarrow \mathfrak{h}_{k} \otimes \mathbb{C}
$$

are $\Sigma_{k}$-equivariant and the latter induces a $\Sigma_{k}$-equivariant homotopy equivalence between $F(\mathbb{C}, k)$ and $\mathfrak{h}_{k} \otimes \mathbb{C}-\bigcup_{i \neq j}\left(L_{i, j} \cap \mathfrak{h}_{k}\right) \otimes \mathbb{C}$.

The arrangement $\left\{L_{i, j} \mid i \neq j\right\}$ in $\mathbb{R}^{k}$ is denoted by $\mathcal{B}_{n}$. Let us denote the induced arrangement in $\mathfrak{h}_{k}$ by $\mathcal{A}_{k-1}$. The purpose of this section is to study the Salvetti complex for this arrangement and compare the skeletal filtration on it to the filtration on $\mathcal{C}_{2}(k)$. 
Note that the chambers of the real complement

$$
\mathbb{R}^{k}-\bigcup_{i \neq j} L_{i, j}
$$

are labeled by elements in $\Sigma_{k}$. For $\sigma \in \Sigma_{k}$, define corresponding points by

$$
\tilde{w}(\sigma)=(\sigma(1), \ldots, \sigma(k)), \quad w(\sigma)=p(\sigma(1), \ldots, \sigma(k)) .
$$

Then each of them belongs to the chamber labeled by $\sigma$. We have

$$
\mathcal{V}^{0}\left(\mathcal{B}_{k}\right)=\left\{\tilde{w}(\sigma) \mid \sigma \in \Sigma_{k}\right\}, \quad \mathcal{V}^{0}\left(\mathcal{A}_{k-1}\right)=\left\{w(\sigma) \mid \sigma \in \Sigma_{k}\right\} .
$$

More generally the faces in the $s$-th stratification (codimension $s$ faces) in the arrangement $\mathcal{B}_{k}$ are labeled by a partition of $\{1, \ldots, k\}$ into ordered $k-s$ nonempty subsets. Recall that we regard a partition as a surjective map

$$
\lambda:\{1, \ldots, k\} \rightarrow\{1, \ldots, k-s\}
$$

for some $s$.

Definition 4.1. Given a partition $\lambda \in \Pi_{k}$, define a face by

$$
F_{\lambda}=\left\{\left(x_{1}, \ldots, x_{k}\right) \in \mathbb{R}^{k} \mid x_{i}<x_{j} \text { if } \lambda(i)<\lambda(j) \text { and } x_{i}=x_{j} \text { if } \lambda(i)=\lambda(j)\right\} .
$$

Lemma 4.2. The faces of $\mathcal{B}_{k}$ are

$$
\mathcal{F}\left(\mathcal{B}_{k}\right)=\left\{F_{\lambda} \mid \lambda \in \Pi_{k}\right\}
$$

For brevity, we denote

$$
\mathfrak{h}_{\lambda}=\mathfrak{h}_{k} \cap F_{\lambda}
$$

Then the faces of $\mathcal{A}_{k-1}$ are

$$
\mathcal{F}\left(\mathcal{A}_{k-1}\right)=\left\{\mathfrak{h}_{\lambda} \mid \lambda \in \Pi_{k}\right\}
$$

We can also use this map $\lambda$ to define a point in each face.

Definition 4.3. For a partition $\lambda \in \Pi_{k}$, define

$$
\tilde{w}(\lambda)=(\lambda(1), \ldots, \lambda(k)), \quad w(\lambda)=p(\tilde{w}(\lambda)) .
$$

Then $\tilde{w}(\lambda)$ and $w(\lambda)$ belong to the face $F_{\lambda}$ and $\mathfrak{h}_{\lambda}$, respectively. Thus we have chosen vertex sets as

$$
\mathcal{V}\left(\mathcal{B}_{k}\right)=\left\{\tilde{w}(\lambda) \mid \lambda \in \Pi_{k}\right\}, \quad \mathcal{V}\left(\mathcal{A}_{k-1}\right)=\left\{w(\lambda) \mid \lambda \in \Pi_{k}\right\}
$$

The ordering of $\Pi_{n}$ corresponding to the ordering of $\mathcal{F}\left(\mathcal{B}_{k}\right)$ is the following. 
Lemma 4.4. Order $\Pi_{k}$ as follows:

$$
\mu \leq \lambda \Leftrightarrow \lambda \text { is a subdivision of } \mu,
$$

in other words

$$
\lambda(i)=\lambda(j) \Rightarrow \mu(i)=\mu(j), \quad \lambda(i)<\lambda(j) \Rightarrow \mu(i) \leq \mu(j) .
$$

Then $\Pi_{k}$ is isomorphic as a poset to $\mathcal{F}\left(\mathcal{B}_{k}\right)$, hence to $\mathcal{F}\left(\mathcal{A}_{k-1}\right)$.

Before we go on to discuss general cases in detail, let us take a look at the simplest case, $k=2$.

Example 4.5. There are three partitions of $\{1,2\}$ :

$$
\Pi_{2}=\{(1 \mid 2),(2 \mid 1),(1,2)\} .
$$

The faces of the arrangement $\mathcal{B}_{2}$ are

$$
\begin{aligned}
& F_{(1 \mid 2)}=\left\{\left(x_{1}, x_{2}\right) \in \mathbb{R}^{2} \mid x_{1}<x_{2}\right\}, \\
& F_{(2 \mid 1)}=\left\{\left(x_{1}, x_{2}\right) \in \mathbb{R}^{2} \mid x_{2}<x_{1}\right\}, \\
& F_{(1,2)}=\left\{\left(x_{1}, x_{2}\right) \in \mathbb{R}^{2} \mid x_{1}=x_{2}\right\}=M_{1,2} .
\end{aligned}
$$

and the faces of $\mathcal{A}_{1}$ are

$$
\mathfrak{h}_{(1 \mid 2)}=F_{(1 \mid 2)} \cap \mathfrak{h}_{2}, \quad \mathfrak{h}_{(2 \mid 1)}=F_{(2 \mid 1)} \cap \mathfrak{h}_{2}, \quad \mathfrak{h}_{(1,2)}=F_{(1,2)} \cap \mathfrak{h}_{2} .
$$

For each face, the assigned vertex is given by

$$
\begin{aligned}
w((1 \mid 2)) & =p(1,2)=(-1 / 2,1 / 2), \quad w((2 \mid 1))=p(2,1)=(1 / 2,-1 / 2), \\
w((1,2)) & =p(1,1)=(0,0),
\end{aligned}
$$

respectively.

We have only 0 -chains and 1 -chains. The 0 -chains are

$$
\mathfrak{h}_{(1,2)}, \mathfrak{h}_{(1 \mid 2)}, \mathfrak{h}_{(2 \mid 1)}
$$

and the 1-chains are

$$
\mathfrak{h}_{(1 \mid 2)}>\mathfrak{h}_{(1,2)}, \quad \mathfrak{h}_{(2 \mid 1)}>\mathfrak{h}_{(1,2)} .
$$

In order to find simplices, we need to determine the face-chamber pairing. By Lemma 2.4, we have

$$
\begin{aligned}
\mathfrak{h}_{(1 \mid 2)} \cdot \mathfrak{h}_{(1 \mid 2)} & =\mathfrak{h}_{(1 \mid 2)}, \\
\mathfrak{h}_{(1 \mid 2)} \cdot \mathfrak{h}_{(2 \mid 1)} & =\mathfrak{h}_{(1 \mid 2)}, \\
\mathfrak{h}_{(2 \mid 1)} \cdot \mathfrak{h}_{(1 \mid 2)} & =\mathfrak{h}_{(2 \mid 1)}, \\
\mathfrak{h}_{(2 \mid 1)} \cdot \mathfrak{h}_{(2 \mid 1)} & =\mathfrak{h}_{(2 \mid 1)} .
\end{aligned}
$$


Since $\left(\mathcal{A}_{2}\right)_{\supset\left|\mathfrak{h}_{(1,2)}\right|}=\mathcal{A}_{2}$, the unique face containing $\mathfrak{h}_{(1 \mid 2)}$ is $\mathfrak{h}_{(1 \mid 2)}$ itself. Since $\mathfrak{h}_{(1,2)}$. $\mathfrak{h}_{(1 \mid 2)}$ is a chamber contained in this $\mathfrak{h}_{(1 \mid 2)}$, we have

$$
\mathfrak{h}_{(1,2)} \cdot \mathfrak{h}_{(1 \mid 2)}=\mathfrak{h}_{(1 \mid 2)}
$$

and for the same reason

$$
\mathfrak{h}_{(1,2)} \cdot \mathfrak{h}_{(2 \mid 1)}=\mathfrak{h}_{(2 \mid 1)}
$$

Thus the 0-simplices, i.e. vertices of the Salvetti complex are

$$
\begin{aligned}
v\left(\mathfrak{h}_{(1,2)}, \mathfrak{h}_{(1,2)} \cdot \mathfrak{h}_{(1 \mid 2)}\right) & =v\left(\mathfrak{h}_{(1,2)}, \mathfrak{h}_{(1 \mid 2)}\right)=w((1,2))+i(w((1 \mid 2))-w((1,2))) \\
& =i w((1 \mid 2)), \\
v\left(\mathfrak{h}_{(1,2)}, \mathfrak{h}_{(1,2)} \cdot \mathfrak{h}_{(2 \mid 1)}\right) & =v\left(\mathfrak{h}_{(1,2)}, \mathfrak{h}_{(2 \mid 1)}\right)=w((1,2))+i(w((2 \mid 1))-w((1,2))) \\
& =i w((2 \mid 1)), \\
v\left(\mathfrak{h}_{(1 \mid 2)}, \mathfrak{h}_{(1 \mid 2)} \cdot \mathfrak{h}_{(1 \mid 2)}\right) & =v\left(\mathfrak{h}_{(1 \mid 2)}, \mathfrak{h}_{(1 \mid 2)}\right)=w((1 \mid 2))+i(w((1 \mid 2))-w((1 \mid 2))) \\
& =w((1 \mid 2)), \\
v\left(\mathfrak{h}_{(2 \mid 1)}, \mathfrak{h}_{(2 \mid 1)} \cdot \mathfrak{h}_{(2 \mid 1)}\right) & =v\left(\mathfrak{h}_{(2 \mid 1)}, \mathfrak{h}_{(2 \mid 1)}\right)=w((2 \mid 1))+i(w((2 \mid 1))-w((2 \mid 1))) \\
& =w((2 \mid 1)) .
\end{aligned}
$$

We also have

$$
\begin{aligned}
v\left(\mathfrak{h}_{(1 \mid 2)}, \mathfrak{h}_{(1 \mid 2)} \cdot \mathfrak{h}_{(2 \mid 1)}\right) & =v\left(\mathfrak{h}_{(1 \mid 2)}, \mathfrak{h}_{(1 \mid 2)}\right)=w((1 \mid 2))+i(w((1 \mid 2))-w((1 \mid 2))) \\
& =w((1 \mid 2)), \\
v\left(\mathfrak{h}_{(2 \mid 1)}, \mathfrak{h}_{(2 \mid 1)} \cdot \mathfrak{h}_{(1 \mid 2)}\right) & =v\left(\mathfrak{h}_{(2 \mid 1)}, \mathfrak{h}_{(2 \mid 1)}\right)=w((2 \mid 1))+i(w((2 \mid 1))-w((2 \mid 1))) \\
& =w((2 \mid 1)) .
\end{aligned}
$$

The 1-simplices are given by

$$
\begin{aligned}
s\left(\mathfrak{h}_{(1 \mid 2)}, \mathfrak{h}_{(1,2)} ; \mathfrak{h}_{(1 \mid 2)}\right) & =\operatorname{Conv}\left(\left\{v\left(\mathfrak{h}_{(1 \mid 2)}, \mathfrak{h}_{(1 \mid 2)} \cdot \mathfrak{h}_{(1 \mid 2)}\right), v\left(\mathfrak{h}_{(1,2)}, \mathfrak{h}_{(1,2)} \cdot \mathfrak{h}_{(1 \mid 2)}\right)\right\}\right) \\
& =\operatorname{Conv}(\{w((1 \mid 2)), i w((1 \mid 2))\}), \\
s\left(\mathfrak{h}_{(1 \mid 2)}, \mathfrak{h}_{(1,2)} ; \mathfrak{h}_{(2 \mid 1)}\right) & =\operatorname{Conv}\left(\left\{v\left(\mathfrak{h}_{(1 \mid 2)}, \mathfrak{h}_{(1 \mid 2)} \cdot \mathfrak{h}_{(2 \mid 1)}\right), v\left(\mathfrak{h}_{(1,2)}, \mathfrak{h}_{(1,2)} \cdot \mathfrak{h}_{(2 \mid 1)}\right)\right\}\right) \\
& =\operatorname{Conv}(\{w((1 \mid 2)), i w((2 \mid 1))\}), \\
s\left(\mathfrak{h}_{(2 \mid 1)}, \mathfrak{h}_{(1,2)} ; \mathfrak{h}_{(1 \mid 2)}\right) & =\operatorname{Conv}\left(\left\{v\left(\mathfrak{h}_{(2 \mid 1)}, \mathfrak{h}_{(2 \mid 1)} \cdot \mathfrak{h}_{(1 \mid 2)}\right), v\left(\mathfrak{h}_{(1,2)}, \mathfrak{h}_{(1,2)} \cdot \mathfrak{h}_{(1 \mid 2)}\right)\right\}\right) \\
& =\operatorname{Conv}(\{w((2 \mid 1)), i w((1 \mid 2))\}), \\
s\left(\mathfrak{h}_{(2 \mid 1)}, \mathfrak{h}_{(1,2)} ; \mathfrak{h}_{(2 \mid 1)}\right) & =\operatorname{Conv}\left(\left\{v\left(\mathfrak{h}_{(2 \mid 1)}, \mathfrak{h}_{(2 \mid 1)} \cdot \mathfrak{h}_{(2 \mid 1)}\right), v\left(\mathfrak{h}_{(1,2)}, \mathfrak{h}_{(1,2)} \cdot \mathfrak{h}_{(2 \mid 1)}\right)\right\}\right) \\
& =\operatorname{Conv}(\{w((2 \mid 1)), i w((2 \mid 1))\}) .
\end{aligned}
$$

It follows that the Salvetti complex $\operatorname{Sal}\left(\mathcal{A}_{1}\right)$ is a 1-dimensional simplicial complex with four vertices $(1 / 2,-1 / 2),(-1 / 2,1 / 2),(i / 2,-i / 2),(-i / 2, i / 2)$ and four edges $[(-1 / 2,1 / 2),(-i / 2, i / 2)], \quad[(-i / 2, i / 2),(1 / 2,-1 / 2)], \quad[(1 / 2,-1 / 2),(i / 2,-i / 2)]$, $[(i / 2,-i / 2),(-i / 2, i / 2)]$. It is the boundary of a square in $\mathfrak{h}_{2}^{\mathbb{C}}$ as is illustrated in the following picture. 


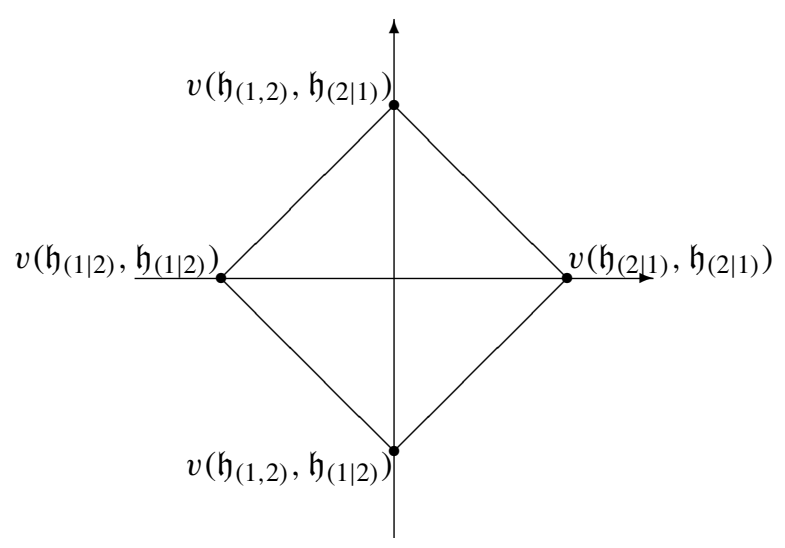

Note that under the composition of the inclusion maps in (10), the vertices $v\left(\mathfrak{h}_{(1 \mid 2)}, \mathfrak{h}_{(1 \mid 2)}\right)$, $v\left(\mathfrak{h}_{(1,2)}, \mathfrak{h}_{(2 \mid 1)}\right), v\left(\mathfrak{h}_{(2 \mid 1)}, \mathfrak{h}_{(2 \mid 1)}\right)$, and $v\left(\mathfrak{h}_{(1,2)}, \mathfrak{h}_{(1 \mid 2)}\right)$ correspond to cubes

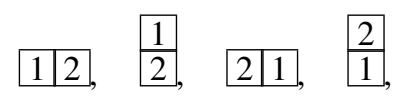

respectively. And we have the following picture:

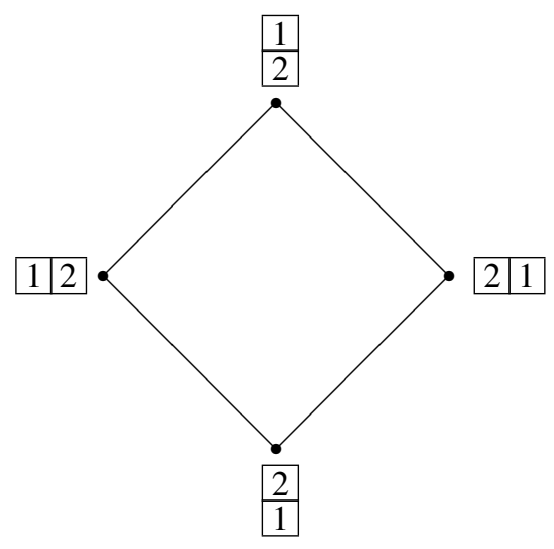

Let us consider the cell structure of $\operatorname{Sal}\left(\mathcal{A}_{1}\right)$. According to Lemma 2.8, 1-cells are

$$
\begin{aligned}
D\left(\mathfrak{h}_{(1,2)}, h_{(1 \mid 2)}\right) & =s\left(\mathfrak{h}_{(1 \mid 2)}, \mathfrak{h}_{(1,2)} ; \mathfrak{h}_{(1 \mid 2)}\right) \cup s\left(\mathfrak{h}_{(2 \mid 1)}, \mathfrak{h}_{(1,2)} ; \mathfrak{h}_{(1 \mid 2)}\right) \\
& =\operatorname{Conv}(\{w((1 \mid 2)), i w((1 \mid 2))\}) \cup \operatorname{Conv}(\{w((2 \mid 1)), i w((1 \mid 2))\}) \\
& =
\end{aligned}
$$




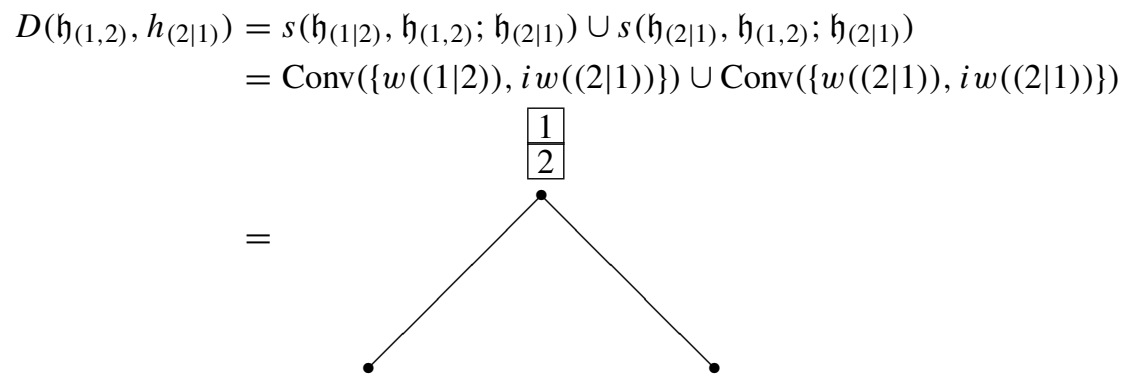

and the 0-cells are

$$
\begin{aligned}
& D\left(\mathfrak{h}_{(1 \mid 2)}, \mathfrak{h}_{(1 \mid 2)}\right)=v\left(\mathfrak{h}_{(1 \mid 2)}, \mathfrak{h}_{(1 \mid 2)}\right)=w((1 \mid 2))=1 \\
& D\left(\mathfrak{h}_{(2 \mid 1)}, \mathfrak{h}_{(2 \mid 1)}\right)=v\left(\mathfrak{h}_{(2 \mid 1)}, \mathfrak{h}_{(2 \mid 1)}\right)=w((2 \mid 1))=2 \\
& \hline
\end{aligned}
$$

Note that the 1-cells are labeled by the cubes \begin{tabular}{l|l|}
\hline & 2 \\
1
\end{tabular} and \begin{tabular}{|l|l|}
\hline \\
2
\end{tabular} in $F_{-1} \mathcal{C}_{2}(2)-F_{-2} \mathcal{C}_{2}(2)$ and the 0-cells are labeled by cubes \begin{tabular}{ll|l|l|l|l|}
\hline & 2
\end{tabular} and $F_{-2} \mathcal{C}_{2}(2)$. Thus the gravity filtration induces a filtration on the Salvetti complex for $\mathcal{A}_{1}$ which coincides with the skeletal filtration up to the shift of filtration by 2 .

Let us return to the general case. For $\lambda \in \Pi_{k}$ and $\sigma \in \Sigma_{k}$, the real parts of the coordinates of the point $v(\lambda, \sigma)$ are determined by $\lambda$ and the imaginary parts are determined by $\sigma$. To be more precise, it is convenient to use the following symbols.

Definition 4.6. For a partition $\lambda \in \Pi_{k}$ of rank $r$ and $\sigma \in \Sigma_{k}$, define a symbol $S(\lambda, \sigma)$ as follows:

1. For each $1 \leq i \leq k-r$, draw vertically stacked squares $S_{i}$ of length $\left|\lambda^{-1}(i)\right|$.

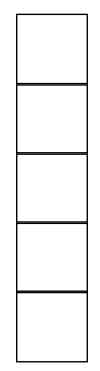

2. Order $\lambda^{-1}(i)$ according to $\sigma$ and label each square in $S_{i}$ from bottom to top by elements in $\lambda^{-1}(i)$. For example, when $\lambda^{-1}(i)=\left\{i_{1}, i_{2}, i_{3}, i_{4}, i_{5}\right\}$ and if these numbers appear in $(\sigma(1), \ldots, \sigma(k))$ in the order

$$
i_{1}, i_{2}, i_{3}, i_{4}, i_{5}
$$

then $S_{i}$ is labeled as 


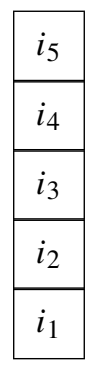

3. Place $S_{1}, \ldots, S_{k-r}$ side by side from left to right. $S(\lambda, \sigma)$ is the resulting picture.

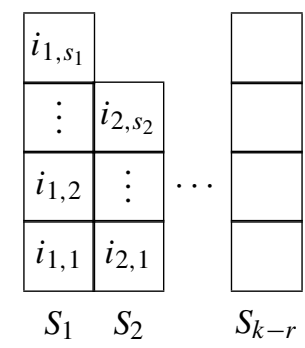

The vertices of the simplicial complex $\operatorname{Sal}\left(\mathcal{A}_{k-1}\right)$ are given by

$$
v\left(\mathfrak{h}_{\lambda}, \mathfrak{h}_{\sigma}\right)=w(\lambda)+i(w(\sigma)-w(\lambda))
$$

for a partition $\lambda$ and a permutation $\sigma \in \Sigma_{k}$ which is a subdivision of $\lambda$.

Lemma 4.7. There is a bijection between the set of vertices $\operatorname{sk}_{0}\left(\operatorname{Sal}\left(\mathcal{A}_{k-1}\right)\right)$ and the set of symbols $\left\{S(\lambda, \sigma) \mid \lambda \in \Pi_{k}, \sigma \in \Sigma_{k}, \lambda \leq \sigma\right\}$.

Definition 4.8. Define a filtration on the vertex $\operatorname{set}^{\operatorname{sk}_{0}}\left(\operatorname{Sal}\left(\mathcal{A}_{k-1}\right)\right)$ by the number of distinct real coordinates:

$$
F_{-s} \operatorname{sk}_{0}\left(\operatorname{Sal}\left(\mathcal{A}_{k-1}\right)\right)=\{w(\lambda)+i(w(\sigma)-w(\lambda))|| \operatorname{Im} \lambda \mid \geq s\} .
$$

The associated filtration on the Salvetti complex by subcomplexes is denoted by

$$
\begin{aligned}
& \emptyset=F_{-k-1} \operatorname{Sal}\left(\mathcal{A}_{k-1}\right) \subset F_{-k} \operatorname{Sal}\left(\mathcal{A}_{k-1}\right) \subset \cdots \\
& \quad \subset F_{-s} \operatorname{Sal}\left(\mathcal{A}_{k-1}\right) \subset F_{-s+1} \operatorname{Sal}\left(\mathcal{A}_{k-1}\right) \subset \cdots \subset F_{-1} \operatorname{Sal}\left(\mathcal{A}_{k-1}\right)=\operatorname{Sal}\left(\mathcal{A}_{k-1}\right) .
\end{aligned}
$$

Note that this is a filtration by $\Sigma_{k}$-subcomplexes.

Lemma 4.9. The composition of the standard homotopy equivalences

$\operatorname{Sal}\left(\mathcal{A}_{k-1}\right) \hookrightarrow \mathfrak{h}_{k} \otimes \mathbb{C}-\bigcup_{i \neq j}\left(L_{i, j} \cap \mathfrak{h}_{k}\right) \otimes \mathbb{C} \hookrightarrow \mathbb{C}^{k}-\bigcup_{i \neq j} L_{i, j} \otimes \mathbb{C}=F(\mathbb{C}, k) \rightarrow \mathcal{C}_{2}(k)$ preserves the filtrations. Furthermore, the vertex $v(\lambda, \sigma)$ is mapped to the symbol $S(\lambda, \sigma)$ which is regarded as an element of $\mathcal{C}_{2}(k)$ in an obvious way. 
Let us denote this composition by

$$
\varphi_{k}: \operatorname{Sal}\left(\mathcal{A}_{k-1}\right) \rightarrow \mathcal{C}_{2}(k)
$$

Let us take a look at the induced map on the subquotients

$$
F_{-s} \operatorname{Sal}\left(\mathcal{A}_{k-1}\right) / F_{-s-1} \operatorname{Sal}\left(\mathcal{A}_{k-1}\right) \rightarrow F_{-s} \mathcal{C}_{2}(k) / F_{-s-1} \mathcal{C}_{2}(k)
$$

Since the space of little 1 -cubes, $\mathcal{C}_{1}(k)$, is $\Sigma_{k}$-equivariantly homotopy equivalent to $\Sigma_{k}$, Proposition 3.8 give us the following description for the subquotients for $\mathcal{C}_{2}(k)$ :

$$
F_{-s} \mathcal{C}_{2}(k) / F_{-s-1} \mathcal{C}_{2}(k) \simeq \bigvee_{\lambda \in \Pi_{k, k-s}}\left(\Sigma_{\lambda}\right)_{+} \wedge P^{\lambda} / \partial P^{\lambda}
$$

where

$$
\Sigma_{\lambda}=\Sigma_{\lambda^{-1}(1), \ldots, \lambda^{-1}(s)}=\Sigma_{\lambda^{-1}(1)} \times \cdots \times \Sigma_{\lambda^{-1}(s)} .
$$

The subquotients for the Salvetti complex can be easily found by noticing that our filtration essentially coincides with the skeletal filtration in Lemma 2.8.

Lemma 4.10. Let $\operatorname{Sal}\left(\mathcal{A}_{k-1}\right)^{(s)}$ denote the s-skeleton of $\operatorname{Sal}\left(\mathcal{A}_{k-1}\right)$ under the cell structure defined in Lemma 2.8. Then

$$
F_{-s} \operatorname{Sal}\left(\mathcal{A}_{k-1}\right)=\operatorname{Sal}\left(\mathcal{A}_{k-1}\right)^{(k-s)}
$$

Corollary 4.11. We have a homeomorphism

$$
\begin{aligned}
F_{-s} \operatorname{Sal}\left(\mathcal{A}_{k-1}\right) / F_{-s-1} & \operatorname{Sal}\left(\mathcal{A}_{k-1}\right) \\
& \cong \bigvee_{\lambda \in \Pi_{k, k-s}}\left\{\sigma \in \Sigma_{k} \mid \lambda \leq \sigma\right\}_{+} \wedge D(\lambda, \sigma) / \partial D(\lambda, \sigma)
\end{aligned}
$$

Note that, given a partition $\lambda$ of rank $k-s$, the set $\left\{\sigma \in \Sigma_{k} \mid \lambda \leq \sigma\right\}$ is in one-to-one correspondence to the set $\Sigma_{\lambda^{-1}(1)} \times \cdots \times \Sigma_{\lambda-1}(s)$. By investigation, we see that the map $\varphi_{k}$ induces a $\Sigma_{k}$-equivariant homotopy equivalence on each subquotient.

Theorem 4.12. $\varphi_{k}$ induces a $\Sigma_{k}$-equivariant homotopy equivalence, for each $s$,

$$
\varphi_{k}: F_{-s} \operatorname{Sal}\left(\mathcal{A}_{k-1}\right) / F_{-s-1} \operatorname{Sal}\left(\mathcal{A}_{k-1}\right) \stackrel{\Sigma_{k}}{\rightarrow} F_{-s} \mathcal{C}_{2}(k) / F_{-s-1} \mathcal{C}_{2}(k)
$$

Recall that the spectral sequence (2) is induced from the stable filtration on $\Omega^{2} \Sigma^{2} X$ defined by the filtration (5) on little 2 -cubes. Thus we obtain the following description of the $E^{1}$-term. 
Corollary 4.13. The $E^{1}$-term of the spectral sequence defined in [Tam94] can be identified as follows:

$$
\begin{aligned}
E_{-s, t}^{1} & =\bigoplus_{k} E_{-s, t}^{1}\left(\mathcal{A}_{k-1}\right) \cong \bigoplus_{k} C_{k-s}\left(\operatorname{Sal}\left(\mathcal{A}_{k-1}\right)\right) \otimes_{\Sigma_{k}} \tilde{h}_{t-k}\left(X^{\wedge k}\right) \\
& \cong \bigoplus_{k} C_{k-s}\left(\operatorname{Sal}\left(\mathcal{A}_{k-1}\right)\right) \otimes_{\Sigma_{k}} \tilde{h}_{t}\left((\Sigma X)^{\wedge k}\right) \\
& \cong \bigoplus_{k} \mathbb{Z}\left\langle[D(\lambda, \sigma)] \mid \lambda \in \Pi_{k, k-s}, \sigma \in \Sigma_{k}, \lambda \leq \sigma\right\rangle \otimes_{\Sigma_{k}} \tilde{h}_{t}\left((\Sigma X)^{\wedge k}\right) .
\end{aligned}
$$

This is an isomorphism of chain complexes.

Thus the $d^{1}$ of the spectral sequence is given by the cell structure of $\operatorname{Sal}\left(\mathcal{A}_{k-1}\right)$. Lemma 2.8 also gives us a concrete description of the first differential. Note that the cells in $\operatorname{Sal}\left(\mathcal{A}_{k-1}\right)$ are labeled by pairs of a partition and a permutation. In order to make an explicit calculation, we use the following notation.

Definition 4.14. We denote a partition $\lambda$ of rank $r$ as a sequence of subsets

$$
\lambda=\left(\lambda^{-1}(1)|\cdots| \lambda^{-1}(k-r)\right)
$$

or as a sequence of numbers separated by vertical lines

$$
\lambda=\left(i_{1,1}, \ldots, i_{1, s_{1}}|\cdots| i_{k-r, 1}, \ldots, i_{k-r, s_{k-r}}\right)
$$

when $\lambda^{-1}(1)=\left\{i_{1,1}, \ldots, i_{1, s_{1}}\right\}, \ldots, \lambda^{-1}(k-r)=\left\{i_{k-r, 1}, \ldots, i_{k-r, s_{k-r}}\right\}$.

The symmetric group $\Sigma_{k}$ acts on the set of partitions $\Pi_{k, r}$. As a representative of each orbit under this action, we may choose a partition which is an order-preserving map when regarded as a map $\lambda:\{1, \ldots, k\} \rightarrow\{1, \ldots, k-r\}$. Let $O_{k, r}$ be the set of order-preserving partitions of rank $r$.

An element $\lambda \in \Pi_{k, r}$ is said to be of type $\left(p_{1}, \ldots, p_{k-r}\right)$ if

$$
\left|\lambda^{-1}(1)\right|=p_{1}, \quad \ldots, \quad\left|\lambda^{-1}(k-r)\right|=p_{k-r} .
$$

The type of $\lambda$ is denoted by $t(\lambda)$.

Corollary 4.15. The $E^{1}$-term is given by

$$
E_{-s, t}^{1} \cong \bigoplus_{n} \mathbb{Z}\left\langle[D(\lambda,(1|\cdots| k))] \mid \lambda \in O_{k, k-s}\right\rangle \otimes \tilde{h}_{t}(\Sigma X)^{\otimes k}
$$

Example 4.16. Suppose $h_{*}(-)$ satisfies the Künneth isomorphism and consider

$$
d_{-1, t}^{1}: E_{-1, t}^{1} \rightarrow E_{-2, t}^{1} .
$$


We have

$$
\begin{aligned}
E_{-1, *}^{1} & \cong \bigoplus_{k} C_{k-1}\left(\operatorname{Sal}\left(\mathcal{A}_{k-1}\right)\right) \otimes_{\Sigma_{k}} \tilde{h}_{*}(\Sigma X)^{\otimes k} \\
& \cong \bigoplus_{k} \mathbb{Z}\left[\Sigma_{k}\right]\langle[D((1, \ldots, k),(1|\cdots| k))]\rangle \otimes \Sigma_{k} \tilde{h}_{t}(\Sigma X)^{\otimes k} \\
& \cong \bigoplus_{k} \mathbb{Z}\langle[D((1, \ldots, k),(1|\cdots| k))]\rangle \otimes \tilde{h}_{t}(\Sigma X)^{\otimes k} \\
E_{-2, *}^{1} & \cong \bigoplus_{k} C_{k-2}\left(\operatorname{Sal}\left(\mathcal{A}_{k-1}\right)\right) \otimes \Sigma_{k} \tilde{h}_{*}(\Sigma X)^{\otimes k} \\
& \cong \bigoplus_{k} \mathbb{Z}\left\langle[D(\lambda,(1|\cdots| k))] \mid \lambda \in O_{k, k-2}\right\rangle \otimes \tilde{h}_{*}(\Sigma X)^{\otimes k}
\end{aligned}
$$

Consider the summand for $k=3$. Under the action of $\Sigma_{3}, \Pi_{3,1}$ has two orbits. One is represented by $(1 \mid 2,3)$ and the other by $(1,2 \mid 3)$. The first differential is a map

$$
\begin{aligned}
& d_{-1, *}^{1}: \mathbb{Z}\langle([D(1,2,3),(1|2| 3)])\rangle \otimes \tilde{h}_{*}(\Sigma X)^{\otimes 3} \\
& \rightarrow \mathbb{Z}\left\langle[D(\lambda,(1|2| 3))] \mid \lambda \in O_{3,1}\right\rangle \otimes \tilde{h}_{*}(\Sigma X)^{\otimes 3} \\
&=\mathbb{Z}\langle[D((1 \mid 2,3),(1|2| 3))],[D((1,2 \mid 3),(1|2| 3))]\rangle \otimes \tilde{h}_{*}(\Sigma X)^{\otimes 3} .
\end{aligned}
$$

For elements $x_{1}, x_{2}, x_{3} \in \tilde{h}_{*}(X)$, let us write the element $\left(\Sigma x_{1}\right) \otimes\left(\Sigma x_{2}\right) \otimes\left(\Sigma x_{3}\right) \in$ $\tilde{h}_{*}(\Sigma X)^{\otimes 3}$ by $\left[x_{1}\left|x_{2}\right| x_{3}\right]$. Then we have

$$
\begin{aligned}
d_{-1, t}^{1}\left([D((1,2,3),(1|2| 3))] \otimes\left[x_{1}\left|x_{2}\right| x_{3}\right]\right) & \\
& =\sum_{\lambda \in \Pi_{3,1}}[D(\lambda, \lambda \circ(1|2| 3))] \otimes_{\Sigma_{3}}\left[x_{1}\left|x_{2}\right| x_{3}\right]
\end{aligned}
$$

in $C_{1}\left(\operatorname{Sal}\left(\mathcal{A}_{2}\right)\right) \otimes_{\Sigma_{3}} \otimes \tilde{h}_{*}(\Sigma X)^{\otimes 3}$, where each $D(\lambda, \lambda \circ(1|2| 3))$ has the orientation induced from $D((1,2,3),(1|2| 3))$.

For $\lambda=\left(i_{1} \mid i_{2}, i_{3}\right) \in \Pi_{3,1}$ with $i_{2}<i_{3}$, let $\sigma=\left(i_{1}\left|i_{2}\right| i_{3}\right) \in \Pi_{3,0}=\Sigma_{3}$ then

$$
\lambda=(1 \mid 2,3) \circ \sigma .
$$

The difference of orientations between $D((1 \mid 2,3),(1|2| 3))$ and $D\left(\left(i_{1} \mid i_{2}, i_{3}\right),(1|2| 3)\right)$ is $\operatorname{sgn}(\sigma)$ where sgn is the sign function

$$
\operatorname{sgn}: \Sigma_{3} \rightarrow\{ \pm 1\}
$$

Then we have

$$
\begin{aligned}
{[D(\lambda, \lambda \circ(1|2| 3))] \otimes_{\Sigma_{3}}\left[x_{1}\left|x_{2}\right| x_{3}\right] } & =\operatorname{sgn}(\sigma)[D((1 \mid 2,3),(1|2| 3))] \cdot \sigma \otimes_{\Sigma_{3}}\left[x_{1}\left|x_{2}\right| x_{3}\right] \\
& =\operatorname{sgn}(\sigma)[D((1 \mid 2,3),(1|2| 3))] \otimes_{\Sigma_{3}}\left[x_{1}\left|x_{2}\right| x_{3}\right] \cdot \sigma
\end{aligned}
$$


and

$$
\begin{aligned}
d_{-1, t}^{1}\left(\left[D((1,2,3),(1|2| 3)] \otimes\left[x_{1}\left|x_{2}\right| x_{3}\right]\right)\right. & \\
= & {[D((1 \mid 2,3),(1|2| 3))] \otimes\left(\sum_{\sigma \in S_{1,2}} \operatorname{sgn}(\sigma)\left[x_{1}\left|x_{2}\right| x_{3}\right] \cdot \sigma\right) } \\
& +[D((1,2 \mid 3),(1|2| 3))] \otimes\left(\sum_{\sigma \in S_{2,1}} \operatorname{sgn}(\sigma)\left[x_{1}\left|x_{2}\right| x_{3}\right] \cdot \sigma\right)
\end{aligned}
$$

where $S_{1,2}$ and $S_{2,1}$ are the sets of $(1,2)$ - and $(2,1)$-shuffles.

For general $n, d_{-1, *}^{1}$ can also be described by $(p, q)$-shuffles.

In order to give a precise description of $d^{1}$, we need to compare orientations of cells in $\operatorname{Sal}\left(\mathcal{A}_{k-1}\right)$. Note that the action of $\Sigma_{k}$ on $\operatorname{Sal}\left(\mathcal{A}_{k-1}\right)$ is induced by permutation of coordinates. For an $s$-cell $D(\lambda, \rho)$, choose an orientation. Each $(s-1)$-cell in the boundary is assigned the orientation induced from $D(\lambda, \rho)$. For two $(s-1)$-cells in the boundary, $D(\mu, \mu \circ \rho)$ and $D\left(\mu^{\prime}, \mu^{\prime} \circ \rho\right)$, if we write

$$
D\left(\mu^{\prime}, \mu^{\prime} \circ \rho\right)=D(\mu, \mu \circ \rho) \cdot \sigma
$$

with $\sigma \in \Sigma_{k}$, the $(s-1)$-cell $D\left(\mu^{\prime}, \mu^{\prime} \circ \rho\right)$ has two orientations, one induced from $D(\lambda, \rho)$ and the other induced from $D(\mu, \mu \circ \rho)$. The difference of the orientations is the determinant of the linear action of $\sigma, \operatorname{sgn}(\sigma)$.

We also need the following notation.

Definition 4.17. An element $\sigma \in \Sigma_{k}$ is called a $\left(p_{1}, \ldots, p_{s}\right)$-shuffle if $p_{1}+\cdots+p_{s}=k$ and

$$
\begin{aligned}
& \sigma(1)<\cdots<\sigma\left(p_{1}\right) \\
& \sigma\left(p_{1}+1\right)<\cdots<\sigma\left(p_{1}+p_{2}\right) \\
& \cdots \\
& \sigma\left(p_{1}+\cdots+p_{s-1}+1\right)<\cdots<\sigma\left(p_{1}+\cdots+p_{s}\right)=\sigma(k) .
\end{aligned}
$$

The set of $\left(p_{1}, \ldots, p_{s}\right)$-shuffles is denoted by $S_{p_{1}, \ldots, p_{s}}$.

Corollary 4.18. For $x_{1}, \ldots, x_{k} \in \tilde{h}_{*}(X)$, denote

$$
\left[x_{1}|\cdots| x_{k}\right]=\left(\Sigma x_{1}\right) \otimes \cdots \otimes\left(\Sigma x_{k}\right) \in \tilde{h}_{*}(\Sigma X)^{\otimes k} .
$$

Then, for $\lambda \in O_{k, k-s}$, the first differential in the spectral sequence is given by

$$
\begin{aligned}
d_{-s, *}^{1}([D(\lambda, & \left.(1|\cdots| k))] \otimes\left[x_{1}|\cdots| x_{k}\right]\right) \\
& =\sum_{\tau \in O_{k, k-s-1}, \lambda<\tau}[D(\tau,(1|\cdots| k))] \otimes\left(\sum_{\rho \in S_{t(\tau)}} \operatorname{sgn}(\rho)\left[x_{1}|\cdots| x_{k}\right] \cdot \rho\right),
\end{aligned}
$$

where $[D(\tau,(1|\cdots| k))]$ has the orientation induced from that of $[D(\lambda,(1|\cdots| k))]$. Recall that $S_{t(\tau)}$ is the set of shuffles of the same type as $\tau$. 
It is worth noting that the first differentials are induced by space-level shuffles

$$
\bigvee_{\lambda \in O_{k, k-s}} S_{(\lambda,(1|\cdots| k))}^{k-s} \wedge(\Sigma X)^{\wedge k} \rightarrow \Sigma\left(\bigvee_{\tau \in O_{k, k-s-1, \lambda<\tau}} S_{(\tau,(1|\cdots| k))}^{n-s-1} \wedge(\Sigma X)^{\wedge k}\right)
$$

where $S_{(\lambda,(1|\cdots| k))}^{k-s}$ and $S_{(\tau,(1|\cdots| k))}^{k-s-1}$ are copies of the spheres $S^{k-s}$ and $S^{k-s-1}$, respectively.

This map may be of some use to study maps between wedge powers of suspended spaces.

\section{Higher order Salvetti complexes}

Björner and Ziegler briefly discussed higher order oriented matroids in $§ 9.4$ of [BZ92]. Let us construct higher order Salvetti complexes for oriented matroids based on their idea.

\subsection{Oriented matroids and real arrangements}

In order to make the construction of the Salvetti complex described in $\$ 2$ higher dimensional, we use oriented matroids. Let us recall the relations between oriented matroids and real arrangements.

Let $\mathcal{A}=\left\{H_{1}, \ldots, H_{n}\right\}$ be a real central arrangement in a vector space $V$. Choosing a normal vector $\boldsymbol{v}_{i}$ in each hyperplane $H_{i}$, we obtain a vector configuration $\mathcal{V}=$ $\left\{\boldsymbol{v}_{1}, \ldots, \boldsymbol{v}_{n}\right\}$ in $V$. Consider the set of linear dependencies of $\mathcal{V}$ :

$$
\begin{aligned}
\operatorname{lin}-\operatorname{dep}(\mathcal{V}) & =\left\{\boldsymbol{\lambda}=\left(\lambda_{1}, \ldots, \lambda_{n}\right) \in \mathbb{R}^{n}-\{\mathbf{0}\} \mid \lambda_{1} \boldsymbol{v}_{1}+\cdots+\lambda_{n} \boldsymbol{v}_{n}=\mathbf{0}\right\} \\
& =\left\{\boldsymbol{\lambda}: \mathcal{V} \rightarrow \mathbb{R} \mid \boldsymbol{\lambda}\left(\boldsymbol{v}_{1}\right) \boldsymbol{v}_{1}+\cdots+\boldsymbol{\lambda}\left(\boldsymbol{v}_{n}\right) \boldsymbol{v}_{n}=\mathbf{0}, \boldsymbol{\lambda} \neq 0\right\}
\end{aligned}
$$

We say a linear dependency $\boldsymbol{\lambda}$ is minimal if it is no longer a linear dependency when any one of the $\lambda_{i}$ 's is replaced with 0 .

By using the sign function sign : $\mathbb{R} \rightarrow\{+1,0,-1\}$ defined by

$$
\operatorname{sign}(x)= \begin{cases}+1, & x>0 \\ 0, & x=0 \\ -1, & x<0\end{cases}
$$

we obtain a map

$$
\operatorname{sign} \circ \lambda: \mathcal{V} \rightarrow\{+1,0,-1\}
$$

The set

$$
\mathcal{C}(\mathcal{V})=\{\operatorname{sign} \circ \boldsymbol{\lambda} \mid \boldsymbol{\lambda} \in \operatorname{lin}-\operatorname{dep}(\mathcal{V}) \text { minimal }\}
$$

is called the set of signed circuits of $\mathcal{V}$. This collection $\mathcal{C}(\mathcal{V})$ of signed circuits on $\mathcal{V}$ is a typical example of an oriented matroid. In order to introduce a general definition of oriented matroid, we regard $+1,0$, and -1 just as symbols and denote the set of three 
elements $\{+1,0,-1\}$ by $S_{1}$. The set $S_{1}$ is equipped with a natural $\mathbb{Z}_{2}$-action. Note that a function on a set $E$ with values in $S_{1}$,

$$
X: E \rightarrow S_{1}
$$

can be regarded as a "signed subset" of $E$, i.e. $X$ determines and is determined by two disjoint subsets of $E$,

$$
X_{+}=X^{-1}(+1), \quad X_{-}=X^{-1}(-1) .
$$

The most fundamental signed subsets are the Kronecker delta functions $\delta_{x}^{+}, \delta_{x}^{-}$defined for $x \in E$ by

$$
\delta_{x}^{+}(y)=\left\{\begin{array}{ll}
+1, & x=y, \\
0, & x \neq y,
\end{array} \quad \delta_{x}^{-}(y)= \begin{cases}-1, & x=y \\
0, & x \neq y\end{cases}\right.
$$

A signed subset $X$ of $E$ can be regarded as a subset of $E^{ \pm}=\left\{\delta_{x}^{+}, \delta_{x}^{-} \mid x \in E\right\}$ satisfying the following disjointness condition:

$$
\delta_{x}^{ \pm} \in X \Rightarrow \delta_{x}^{\mp} \notin X .
$$

For simplicity, we denote $\delta_{x}^{ \pm}$by $\pm x$.

Remark 5.1. The adjoint

$$
\operatorname{ad}(\delta): E \hookrightarrow \mathcal{P}(E)
$$

of the usual delta function

$$
\delta: E \times E \rightarrow\{0,1\}
$$

embeds $E$ in $\operatorname{Map}(E,\{0,1\})=\mathcal{P}(E)$ as characteristic functions.

Analogously, the signed delta functions

$$
\delta^{+}: E \times E \rightarrow S_{1}, \quad \delta^{-}: E \times E \rightarrow S_{1}
$$

defined by

$$
\delta^{ \pm}(x, y)= \begin{cases} \pm 1, & x=y \\ 0, & x \neq y\end{cases}
$$

induce embeddings

$$
\operatorname{ad}\left(\delta^{+}\right): E \hookrightarrow \operatorname{Map}\left(E, S_{1}\right), \quad \operatorname{ad}\left(\delta^{-}\right): E \hookrightarrow \operatorname{Map}\left(E, S_{1}\right) .
$$

The set $E^{ \pm}$is nothing other than the union of the images of these maps,

$$
E^{ \pm}=\operatorname{ad}\left(\delta^{+}\right)(E) \cup \operatorname{ad}\left(\delta^{-}\right)(E) .
$$

Note that the action of $\mathbb{Z}_{2}$ on $S_{1}$ induces an action on $E^{ \pm}$. 
Definition 5.2. Given a set $E$, let

$$
*: E^{ \pm} \rightarrow E^{ \pm}
$$

be the involution defined by $( \pm x)^{*}=\mp x$. A signed subset of $E$ is a subset $X$ of $E^{ \pm}$with

$$
X \cap X^{*}=\emptyset \text {. }
$$

The set of signed subsets of $E$ is denoted by $\mathcal{P}_{ \pm}(E)$.

The following is a definition of oriented matroid in terms of subsets of $E^{ \pm}$given in [GR89]. It is essentially identical to the definition by "circuit axioms" in [BLVS $\left.{ }^{+} 99\right]$.

Definition 5.3. An oriented matroid on a set $E$ is a pair $\mathcal{M}=(E, \mathcal{C})$, where $\mathcal{C} \subset$ $\mathcal{P}_{ \pm}(E)-\{\emptyset\}$ is a collection of nonempty signed subsets of $E$ satisfying the following conditions:

1. $X \in \mathcal{C} \Rightarrow X^{*} \in \mathcal{C}$,

2. $X_{1}, X_{2} \in \mathcal{C}$ and $X_{1} \subset X_{2} \cup X_{2}^{*} \Rightarrow X_{1}=X_{2}$ or $X_{1}=X_{2}^{*}$,

3. $X_{1}, X_{2} \in \mathcal{C}, e \in X_{1} \cap X_{2}^{*}$, and $X_{1} \neq X_{2}^{*} \Rightarrow$ there exists $Y \in \mathcal{C}$ such that

$$
Y \subset\left(X_{1} \cup X_{2}\right)-\left\{e, e^{*}\right\}
$$

Example 5.4. Given a vector configuration $\mathcal{V}=\left\{\boldsymbol{v}_{1}, \ldots, \boldsymbol{v}_{n}\right\}$ in a real vector space $V$, the pair $\mathcal{M}(\mathcal{V})=(\mathcal{V}, \mathcal{C}(\mathcal{V}))$ is an oriented matroid.

For a real hyperplane arrangement $\mathcal{A}$, the oriented matroid of the normal vector configuration $\mathcal{M}(\mathcal{V})$ is independent of the choice of a normal vector configuration and is denoted by $\mathcal{M}(\mathcal{A})$. And the set of signed circuits is denoted by $\mathcal{C}(\mathcal{A})$.

Combinatorial structures of a real hyperplane arrangement $\mathcal{A}$ can be described in terms of the associated oriented matroid. For example, a face $F$ of $\mathcal{A}$ can be regarded as a function

$$
\tau_{F}: \mathcal{V}^{ \pm} \rightarrow S_{1}
$$

by

$$
\tau_{F}(\boldsymbol{a})= \begin{cases}+1, & F \subset\{\boldsymbol{x} \mid\langle\boldsymbol{a}, \boldsymbol{x}\rangle>0\}, \\ 0, & F \subset\{\boldsymbol{x} \mid\langle\boldsymbol{a}, \boldsymbol{x}\rangle=0\}, \\ -1, & F \subset\{\boldsymbol{x} \mid\langle\boldsymbol{a}, \boldsymbol{x}\rangle<0\} .\end{cases}
$$

The following is a necessary and sufficient condition for such a function to be associated with a face:

Lemma 5.5 (Gel'fand-Rybnikov). Let $\mathcal{A}$ be a real central hyperplane arrangement and $\mathcal{V}$ be a vector configuration of normal vectors. For a function $\tau: \mathcal{V}^{ \pm} \rightarrow S_{1}$, there exists a face $F$ with $\tau=\tau_{F}$ if and only if the following conditions hold:

1. $\tau( \pm \boldsymbol{a})= \pm \tau(\boldsymbol{a})$, i.e. $\tau \in \operatorname{Map}_{\mathbb{Z}_{2}}\left(\mathcal{V}^{ \pm}, S_{1}\right)$. 
2. For any signed circuit $X \in \mathcal{C}(\mathcal{A})$,

$$
\tau(X)=\{0\} \quad \text { or } \tau(X) \supset\{+1,-1\} .
$$

Thus we have a one-to-one correspondence between $\mathcal{L}(\mathcal{A})$ and the set

$$
\left\{\tau \in \operatorname{Map}_{\mathbb{Z}_{2}}\left(\mathcal{V}^{ \pm}, S_{1}\right) \mid \tau(X)=\{0\} \text { or } \tau(X) \supset\{+1,-1\} \text { for } X \in \mathcal{C}(\mathcal{A})\right\} .
$$

The poset structure on $\mathcal{L}(\mathcal{A})$ induces a poset structure on this set.

\section{Lemma 5.6. For}

$$
\varphi, \psi \in\left\{\tau \in \operatorname{Map}_{\mathbb{Z}_{2}}\left(\mathcal{V}^{ \pm}, S_{1}\right) \mid \tau(X)=\{0\} \text { or } \tau(X) \supset\{+1,-1\} \text { for } X \in \mathcal{C}\right\},
$$

$\varphi \leq \psi$ if and only if $\varphi(\boldsymbol{a}) \neq 0 \Rightarrow \psi(\boldsymbol{a})=\varphi(\boldsymbol{a})$.

Definition 5.7. For an oriented matroid $\mathcal{M}=(E, \mathcal{C})$, define

$$
\mathcal{L}(\mathcal{M})=\left\{\tau \in \operatorname{Map}_{\mathbb{Z}_{2}}\left(E^{ \pm}, S_{1}\right) \mid \tau(X)=\{0\} \text { or } \tau(X) \supset\{+1,-1\} \text { for } X \in \mathcal{C}\right\} .
$$

This is regarded as a poset with the following ordering: $\varphi \leq \psi$ if and only if

$$
\varphi(\boldsymbol{a}) \neq 0 \Rightarrow \psi(\boldsymbol{a})=\varphi(\boldsymbol{a}) .
$$

Elements in $\mathcal{L}(\mathcal{M})$ are called faces of $\mathcal{M}$ and a face $\varphi$ is called a chamber if $\varphi(\boldsymbol{a}) \neq 0$ for any $a \in E^{ \pm}$. The set of chambers is denoted by $\mathcal{L}^{(0)}(\mathcal{M})$.

Remark 5.8. For a real central arrangement $\mathcal{A}$ in a vector space $V$, we have a homotopy equivalence

$$
\mathcal{L}^{(0)}(\mathcal{A}) \simeq V-\bigcup_{L \in A} L
$$

if $\mathcal{L}^{(0)}(\mathcal{A})$ is regarded as a discrete 0 -dimensional complex.

An important operation on faces of an oriented matroid is the following matroid product, which corresponds to pairings in Proposition 2.3.

Definition 5.9. For $\varphi, \psi \in \operatorname{Map}\left(E, S_{1}\right)$, define $\varphi \circ \psi \in \operatorname{Map}\left(E, S_{1}\right)$ by

$$
\begin{aligned}
(\varphi \circ \psi)(e) & = \begin{cases}\psi(e), & \varphi(e) \leq \psi(e), \\
\varphi(e), & \text { otherwise },\end{cases} \\
& = \begin{cases}\varphi(e), & \varphi(e) \neq 0, \\
\psi(e), & \varphi(e)=0 .\end{cases}
\end{aligned}
$$

Lemma 5.10. The matroid product has the following properties: for $F_{1}, F_{2} \in \mathcal{L}(\mathcal{M})$,

1. $F_{1} \leq F_{1} \circ F_{2}$,

2. if $F_{2} \in \mathcal{L}^{(0)}(\mathcal{M})$ then $F_{1} \circ F_{2} \in \mathcal{L}^{(0)}(\mathcal{M})$,

3. if $F_{1} \leq F_{2}$ then $F_{1} \circ F_{2}=F_{2}$,

4. $F_{1} \circ 0=F_{1}$. 
It is well-known that the definition of oriented matroid can be given in terms of faces (covectors). In order to describe the condition, it is convenient to introduce the following.

Definition 5.11. For $\sigma, \tau \in \operatorname{Map}_{\mathbb{Z}_{2}}\left(E^{ \pm}, S_{1}\right)$, define $S(\sigma, \tau) \subset E^{ \pm}$by

$$
S(\sigma, \tau)=\left\{x \in E^{ \pm} \mid \sigma(x)=\tau(x)^{*} \neq 0\right\} .
$$

This is called the separation set of $\sigma$ and $\tau$.

We say $\sigma$ is orthogonal to $\tau$ and write $\sigma \perp \tau$ if $S(\sigma, \tau)$ and $S\left(\sigma, \tau^{*}\right)$ are both empty or both nonempty.

The next proposition is called the "covector axiom" in [BLVS +99$]$.

Proposition 5.12. A subset $\mathcal{L}$ of $\operatorname{Map}_{\mathbb{Z}_{2}}\left(E^{ \pm}, S_{1}\right)$ is the set of faces of an oriented matroid on $E$ if and only if it satisfies the following conditions:

1. $0 \in \mathcal{L}$,

2. $\tau \in \mathcal{L} \Rightarrow-\tau \in \mathcal{L}$,

3. for $\tau_{1}, \tau_{2} \in \mathcal{L}, \tau_{1} \circ \tau_{2} \in \mathcal{L}$,

4. for $\tau_{1}, \tau_{2} \in \mathcal{L}$ and $x \in S\left(\tau_{1}, \tau_{2}\right)$, there exists $\tau_{3} \in \mathcal{L}$ such that

(a) $\tau_{3}(x)=0$,

(b) $\tau_{3}(y)=\left(\tau_{1} \circ \tau_{2}\right)(y)=\left(\tau_{2} \circ \tau_{1}\right)(y)$ for all $y \notin S\left(\tau_{1}, \tau_{2}\right)$.

In other words, a set $\mathcal{L}$ of signed subsets satisfying the above conditions determines a set of signed circuits and vice versa. Note that $\mathcal{P}_{ \pm}(E)$ can be regarded as a subset of $\operatorname{Map}_{\mathbb{Z}_{2}}\left(E^{ \pm},\{+1,0,-1\}\right)$ by identifying $X \in \mathcal{P}_{ \pm}(E)$ with

$$
\delta_{X}(x)= \begin{cases}1, & x \in X, \\ -1, & x \in-X, \\ 0, & \text { otherwise. }\end{cases}
$$

Under this identification, the definition of the set of faces is given as follows:

Proposition 5.13. Let $\mathcal{M}=(E, \mathcal{C})$ be an oriented matroid. Then the set of faces is given by

$$
\mathcal{L}=\left\{\tau \in \operatorname{Map}_{\mathbb{Z}_{2}}\left(E^{ \pm}, S_{1}\right) \mid \tau \perp \varphi \text { for all } \varphi \in \mathcal{C}\right\}
$$

\subsection{Higher order Salvetti complexes for oriented matroids}

In this section, we recall the definition of oriented $\ell$-matroid and the $\ell$-dimensionalization of an (ordinary) oriented matroid due to Björner and Ziegler [BZ92]. It was pointed out by the referee that an analogous construction was given by De Concini and Salvetti in [DCS00] without referring to higher order oriented matroids. It should be noted, however, that these constructions give essentially the same cell complex models for the complement of the $\ell$-dimensionalization of a real central arrangement.

In order to understand these constructions, let us first recall the definition of the Salvetti complex for an oriented matroid. The following description is due to Gel'fand and Rybnikov [GR89]. 
Definition 5.14. Let $S_{2}$ be the set of five elements $S_{2}=\left\{0, e_{1},-e_{1}, e_{2},-e_{2}\right\}$. We define a partial order on $S_{2}$ by the following rule:

$$
0< \pm e_{1}< \pm e_{2} .
$$

For an oriented matroid $\mathcal{M}=(E, \mathcal{C})$, define

$$
\mathcal{L}^{(1)}(\mathcal{M})=\left\{\varphi \in \operatorname{Map}_{\mathbb{Z}_{2}}\left(E^{ \pm}, S_{2}-\{0\}\right) \mid \varphi(X) \subset\left\{ \pm e_{1}\right\} \text { or } \varphi(X) \supset\left\{ \pm e_{2}\right\} \text { for } X \in \mathcal{C}\right\} .
$$

Remark 5.15. The elements of the set $S_{2}$ are just symbols, but we may regard $\left\{e_{1}, e_{2}\right\}$ as the standard orthonormal basis of $\mathbb{R}^{2} . S_{2}$ is considered to be equipped with a $\mathbb{Z}_{2}$-action by changing signs.

The following observation is due to Gel'fand and Rybnikov, but we include a proof in order to help the reader understand an analogous fact for higher-order versions.

Lemma 5.16. The set $\mathcal{L}^{(1)}(\mathcal{M})$ is in one-to-one correspondence with the set of pairs

$$
\left\{(F, C) \in \mathcal{L}(\mathcal{M}) \times \mathcal{L}^{(0)}(\mathcal{M}) \mid F \leq C\right\} .
$$

Proof. In order to define a bijection, take $(F, C)$ in $\mathcal{L}(\mathcal{M}) \times \mathcal{L}^{(0)}(\mathcal{M})$ with $F \leq C$. We use the auxiliary $\mathbb{Z}_{2}$-equivariant maps

$$
s_{1}:\{ \pm 1,0\} \rightarrow\left\{ \pm e_{1}, 0\right\}, \quad s_{2}:\{ \pm 1,0\} \rightarrow\left\{ \pm e_{2}, 0\right\}
$$

defined by

$$
\begin{array}{ll}
s_{1}(1)=e_{1}, & s_{2}(1)=e_{2}, \\
s_{1}(0)=0, & s_{2}(0)=0 .
\end{array}
$$

For a face $F$, define $F \otimes e_{i}$ by

$$
F \otimes e_{i}=s_{i} \circ F: E \rightarrow S_{2} .
$$

For maps $F_{1}, F_{2}: E \rightarrow S_{2}$ we extend the matroid product as follows:

$$
\left(F_{1} \circ F_{2}\right)(x)= \begin{cases}F_{2}(x), & F_{2}(x)>F_{1}(x), \\ F_{1}(x), & \text { otherwise. }\end{cases}
$$

If $C$ is a chamber we obtain an element $\left(F \otimes e_{1}\right) \circ\left(C \otimes e_{2}\right) \in \mathcal{L}^{(1)}(\mathcal{M})$.

In order to see that the correspondence

$$
(F, C) \mapsto\left(F \otimes e_{1}\right) \circ\left(C \otimes e_{2}\right)
$$

is a bijection, we need the $\mathbb{Z}_{2}$-equivariant maps

$$
\pi_{1}:\left\{ \pm e_{1}, \pm e_{2}\right\} \rightarrow\{ \pm 1,0\}, \quad \pi_{2}:\left\{ \pm e_{1}, \pm e_{2}\right\} \rightarrow\{ \pm 1,0\}
$$

defined by

$$
\begin{array}{ll}
\pi_{1}\left(e_{1}\right)=1, & \pi_{2}\left(e_{1}\right)=1, \\
\pi_{1}\left(e_{2}\right)=1, & \pi_{2}\left(e_{2}\right)=0 .
\end{array}
$$


Note that

$$
\begin{array}{ll}
\pi_{1}\left(F \otimes e_{1}\right)=F, & \pi_{2}\left(F \otimes e_{1}\right)=F, \\
\pi_{1}\left(F \otimes e_{2}\right)=F, & \pi_{2}\left(F \otimes e_{2}\right)=0,
\end{array}
$$

and

$$
\begin{aligned}
& \pi_{1}\left(\left(F \otimes e_{1}\right) \circ\left(C \otimes e_{2}\right)\right)=\pi_{1}\left(F \otimes e_{1}\right) \circ \pi_{1}\left(C \otimes e_{2}\right)=F \circ C=C, \\
& \pi_{2}\left(\left(F \otimes e_{1}\right) \circ\left(C \otimes e_{2}\right)\right)=\pi_{2}\left(F \otimes e_{1}\right) \circ \pi_{2}\left(C \otimes e_{2}\right)=F \circ 0=F .
\end{aligned}
$$

This completes the proof.

Definition 5.17. Define a partial order on $\mathcal{L}^{(1)}(\mathcal{M})$ as follows: $\varphi \leq \psi$ if and only if

$$
\begin{aligned}
\varphi(\boldsymbol{a})=e_{1} & \Rightarrow \psi(\boldsymbol{a})=e_{1} \text { or } \pm e_{2}, \\
\varphi(\boldsymbol{a})=-e_{1} & \Rightarrow \psi(\boldsymbol{a})=-e_{1} \text { or } \pm e_{2}, \\
\varphi(\boldsymbol{a})=e_{2} & \Rightarrow \psi(\boldsymbol{a})=e_{2}, \\
\varphi(\boldsymbol{a})=-e_{2} & \Rightarrow \psi(\boldsymbol{a})=-e_{2} .
\end{aligned}
$$

In other words, the ordering on $\mathcal{L}^{(1)}(\mathcal{M})$ is induced from the ordering on $S_{2}=$ $\left\{0, \pm e_{1}, \pm e_{2}\right\}$.

Definition 5.18. The first order Salvetti complex $\operatorname{Sal}^{(1)}(\mathcal{M})$ is the (geometric realization of the) order complex of $\mathcal{L}^{(1)}(\mathcal{M})$.

As we have seen in the proof of Lemma $5.16, \mathcal{L}^{(1)}(\mathcal{M})$ can be regarded as a subposet of $\left(\mathcal{L}(\mathcal{M}) \otimes e_{1}\right) \circ\left(\mathcal{L}(\mathcal{M}) \otimes e_{2}\right)$. The notion of oriented 2-matroid was introduced by Björner and Ziegler in [BZ92] by abstracting the properties of this poset. At the end of their paper, they also introduced the notion of oriented $\ell$-matroid for $\ell \geq 1$.

We simplify their definition a little and introduce the notion of symmetric oriented $\ell$-matroid.

Definition 5.19. Let

$$
S_{\ell}=\left\{0, e_{1},-e_{1}, \ldots, e_{\ell},-e_{\ell}\right\}
$$

be equipped with the following partial order:

$$
\begin{aligned}
0 & <e_{1},-e_{1}, \\
e_{1} & <e_{2},-e_{2}, \\
-e_{1} & <e_{2},-e_{2}, \\
& \vdots \\
e_{\ell-1} & <e_{\ell},-e_{\ell}, \\
-e_{\ell-1} & <e_{\ell},-e_{\ell},
\end{aligned}
$$

and the obvious $\mathbb{Z}_{2}$-action.

A signed $\ell$-vector on a set $E$ is a $\mathbb{Z}_{2}$-equivariant map

$$
\varphi: E^{ \pm} \rightarrow S_{\ell}
$$


For signed $\ell$-vectors $\varphi$ and $\psi$, define the matroid product by

$$
(\varphi \circ \psi)(x)= \begin{cases}\psi(x), & \psi(x)>\varphi(x) \\ \varphi(x), & \text { otherwise }\end{cases}
$$

and the separation set by

$$
S(\varphi, \psi)=\left\{x \in E^{ \pm} \mid \varphi(x)=\psi(x)^{*} \neq 0\right\} .
$$

Note that $S_{\ell}$ is equipped with a natural $\mathbb{Z}_{2}$-equivariant $\Sigma_{\ell}$-action. This naturally leads us to the notion of symmetric oriented $\ell$-matroid.

Definition 5.20. A symmetric oriented $\ell$-matroid is a pair $\mathcal{M}=(E, \mathcal{L})$ of a set $E$ and $\mathcal{L} \subset \operatorname{Map}_{\mathbb{Z}_{2}}\left(E^{ \pm}, S_{\ell}\right)$ satisfying the following conditions:

1. $0 \in \mathcal{L}$,

2. $F \in \mathcal{L} \Rightarrow-F \in \mathcal{L}$,

3. $F \in \mathcal{L} \Rightarrow \sigma \cdot F \in \mathcal{L}$ for any $\sigma \in \Sigma_{\ell}$,

4. $F_{1}, F_{2} \in \mathcal{L} \Rightarrow F_{1} \circ F_{2} \in \mathcal{L}$,

5. $F_{1}, F_{2} \in \mathcal{L}, x \in S\left(F_{1}, F_{2}\right) \Rightarrow \exists F_{3} \in \mathcal{L}$ such that $F_{3}(x)<F_{1}(x), F_{2}(x)$ and

$$
F_{3}(y)=\left(F_{1} \circ F_{2}\right)(y)=\left(F_{2} \circ F_{1}\right)(y) \text { for } y \notin S\left(F_{1}, F_{2}\right) .
$$

Remark 5.21. A symmetric oriented $\ell$-matroid is a special case of an oriented $\ell$-matroid in the sense of Björner and Ziegler.

There is a natural way to construct a symmetric oriented $\ell$-matroid from an oriented (1-)matroid.

Definition 5.22. For $1 \leq i \leq \ell$, define a $\mathbb{Z}_{2}$-equivariant map

$$
s_{i}:\{ \pm 1,0\} \rightarrow\left\{ \pm e_{i}, 0\right\} \hookrightarrow S_{\ell}
$$

by

$$
s_{i}(1)=e_{i}, \quad s_{i}(0)=0 .
$$

Lemma 5.23. Let $\mathcal{M}$ be an oriented (1-)matroid on a set $E$. For a face $F \in \mathcal{L}(\mathcal{M})$ and $1 \leq i \leq \ell$, define $F \otimes e_{i} \in \operatorname{Map}_{\mathbb{Z}_{2}}\left(E^{ \pm}, S_{\ell}\right)$ by

$$
\left(F \otimes e_{i}\right)(x)=s_{i}(F(x)) .
$$

For simplicity, denote

$$
\mathcal{L}(\mathcal{M}) \otimes \mathbb{R}^{\ell}=\left(\mathcal{L}(\mathcal{M}) \otimes e_{1}\right) \circ \cdots \circ\left(\mathcal{L}(\mathcal{M}) \otimes e_{\ell}\right)
$$

Then $\left(E, \mathcal{L}(\mathcal{M}) \otimes \mathbb{R}^{\ell}\right)$ is a symmetric oriented $\ell$-matroid. 
Proof. The first and the second conditions are obviously satisfied. The third and the fourth conditions follow from the fact that

$$
\begin{aligned}
& \left(F \otimes e_{i}\right) \circ\left(F^{\prime} \otimes e_{j}\right)=\left(F^{\prime} \otimes e_{j}\right) \circ\left(F \otimes e_{i}\right) \quad \text { for } i \neq j, \\
& \left(F \otimes e_{i}\right) \circ\left(F^{\prime} \otimes e_{i}\right)=\left(F \circ F^{\prime}\right) \otimes e_{i} .
\end{aligned}
$$

The final condition follows from the corresponding condition for the covector axiom for oriented 1-matroids.

It is useful to identify elements of $\mathcal{L}(\mathcal{M}) \otimes \mathbb{R}^{\ell}$ with a sequence of faces of $\mathcal{M}$.

Lemma 5.24. For an oriented 1 -matroid $\mathcal{M}$, the $\operatorname{set} \mathcal{L}(\mathcal{M}) \otimes \mathbb{R}^{\ell}$ can be identified with

$$
\left\{\left(F_{1}, \ldots, F_{\ell}\right) \in \mathcal{L}(M)^{\ell} \mid F_{1} \leq \cdots \leq F_{\ell}\right\} .
$$

Proof. For $0 \leq i \leq \ell$, define a $\mathbb{Z}_{2}$-equivariant map $\pi_{i}: S_{\ell} \rightarrow\{ \pm 1,0\}$ by

$$
\pi_{i}\left(e_{j}\right)= \begin{cases}0, & j<i \\ 1, & j \geq i\end{cases}
$$

Define a map

$$
c:\left\{\left(F_{1}, \ldots, F_{\ell}\right) \in \mathcal{L}(M)^{k} \mid F_{1} \leq \cdots \leq F_{\ell}\right\} \rightarrow \mathcal{L}(\mathcal{M}) \otimes \mathbb{R}^{\ell}
$$

by

$$
c\left(F_{1}, \ldots, F_{\ell}\right)=\left(F_{1} \otimes e_{1}\right) \circ \cdots \circ\left(F_{\ell} \otimes e_{\ell}\right) .
$$

The inverse to this map is given by $p(F)=\left(\pi_{1} \circ F, \ldots, \pi_{\ell} \circ F\right)$.

Definition 5.25. For a symmetric oriented $\ell$-matroid $\mathcal{M}=(E, \mathcal{L})$, define

$$
\mathcal{L}^{(0)}=\{\varphi \in \mathcal{L} \mid \varphi(x) \neq 0 \text { for all } x \in E\} .
$$

An element of $\mathcal{L}^{(0)}$ is called a tope or a chamber.

Example 5.26. If $\mathcal{M}$ is an oriented 1-matroid on a set $E$, then $\left(E, \mathcal{L}(\mathcal{M}) \otimes \mathbb{R}^{2}\right)$ is a symmetric oriented 2-matroid. It is easy to see that

$$
\left(\mathcal{L}(\mathcal{M}) \otimes \mathbb{R}^{2}\right)^{(0)}=\mathcal{L}^{(1)}(\mathcal{M}) .
$$

Definition 5.27. For an oriented 1-matroid $\mathcal{M}$, let us denote

$$
\mathcal{L}^{(\ell)}(\mathcal{M})=\left(\mathcal{L}(\mathcal{M}) \otimes \mathbb{R}^{\ell+1}\right)^{(0)} .
$$

This set is regarded as a subposet of $\mathcal{L}(\mathcal{M}) \otimes \mathbb{R}^{\ell+1}$, which has a poset structure induced from $S_{\ell+1}$.

The $\ell$-th order Salvetti complex of $\mathcal{M}$ is the (geometric realization of the) order complex, or the classifying space of this poset,

$$
\operatorname{Sal}^{(\ell)}(\mathcal{M})=\left|\Delta\left(\mathcal{L}^{(\ell)}(\mathcal{M})\right)\right| .
$$


Lemma 5.28. The set $\mathcal{L}^{(\ell)}(\mathcal{M})$ can be identified with

$$
\left\{\left(F_{\ell}, \ldots, F_{1}, C\right) \in \mathcal{L}(M)^{\ell} \times \mathcal{L}^{(0)}(\mathcal{M}) \mid F_{\ell} \leq \cdots \leq F_{1} \leq C\right\} .
$$

Let $\mathcal{A}$ be a real central hyperplane arrangement in a vector space $V$. The $\ell$-th order Salvetti complex for $\mathcal{M}(\mathcal{A})$ is denoted by $\operatorname{Sal}^{(\ell)}(\mathcal{A})$.

$\mathrm{Sal}^{(\ell)}(\mathcal{A})$ can be embedded in $V \otimes \mathbb{R}^{\ell+1}-\bigcup_{L \in \mathcal{A}} L \otimes \mathbb{R}^{\ell+1}$ as follows: we choose an interior point $v(F) \in V$ in each face $F$ of the stratification of $V$ by $\mathcal{A}$ and define, for $\varphi \in \mathcal{L}^{(k)}(\mathcal{A})$

$v(\varphi)=v\left(\pi_{0} \circ \varphi\right) \otimes \boldsymbol{e}_{0}+\left(v\left(\pi_{0} \circ \varphi\right)-v\left(\pi_{1} \circ \varphi\right)\right) \otimes \boldsymbol{e}_{1}+\cdots+\left(v\left(\pi_{0} \circ \varphi\right)-v\left(\pi_{\ell} \circ \varphi\right)\right) \otimes \boldsymbol{e}_{\ell}$,

where $\left\{e_{0}, \ldots, e_{\ell}\right\}$ is the standard orthonormal basis for $\mathbb{R}^{\ell+1}$.

$\mathrm{Sal}^{(\ell)}(\mathcal{M})$ is designed to extend the homotopy equivalence

$$
\operatorname{Sal}^{(1)}(\mathcal{A}) \simeq V \otimes \mathbb{R}^{2}-\bigcup_{L \in \mathcal{A}} L \otimes \mathbb{R}^{2}
$$

for real central arrangements as follows.

Theorem 5.29. The map

$$
v: \operatorname{sk}_{0}\left(\operatorname{Sal}^{(\ell)}(\mathcal{A})\right)=\mathcal{L}^{(\ell)}(\mathcal{A}) \hookrightarrow V \otimes \mathbb{R}^{\ell+1}
$$

induces an embedding

$$
v: \operatorname{Sal}^{(\ell)}(\mathcal{A}) \hookrightarrow \mathbb{R}^{\ell+1}-\bigcup_{L \in \mathcal{A}} L \otimes \mathbb{R}^{\ell+1}
$$

as a deformation retract.

This fact seems to be known to Björner and Ziegler [BZ92]. The proof requires no novelty. The proof of the analogous fact in [BZ92] works with obvious modifications. We include a sketch of proof in order to be self-contained.

The idea of Björner and Ziegler is to identify $\operatorname{Sal}^{(k)}(\mathcal{A})$ as the (deformation retract of the) complement of the link poset in $\left|\Delta\left(\mathcal{L}(\mathcal{A}) \otimes \mathbb{R}^{\ell+1}-\{0\}\right)\right|$.

Lemma 5.30. For a real essential central arrangement $\mathcal{A}$ in $V$, the cell complex $\left|\Delta\left(\mathcal{L}(\mathcal{A}) \otimes \mathbb{R}^{\ell+1}-\{0\}\right)\right|$ is homeomorphic to the unit sphere $S\left(V \otimes \mathbb{R}^{\ell+1}\right)$.

Definition 5.31. Define a subposet of $\mathcal{L}(\mathcal{A}) \otimes \mathbb{R}^{\ell+1}-\{0\}$ by

$$
\mathcal{K}_{\text {link }}\left(\mathcal{A} \otimes \mathbb{R}^{\ell+1}\right)=\left\{\varphi \in \mathcal{L}(\mathcal{A}) \otimes \mathbb{R}^{\ell+1}-\{0\} \mid \varphi(e)=0 \text { for some } e\right\} .
$$

This is the complement of $\mathcal{L}^{(\ell)}(\mathcal{A})$ in $\mathcal{L}(\mathcal{A}) \otimes \mathbb{R}^{\ell+1}-\{0\}$.

The following is a well-known fact and can be found in [BLVS ${ }^{+}$99, Lemma 4.7.27].

Lemma 5.32. Let $P$ be a poset and $Q$ a subposet. Then $|\Delta(Q)|$ is a deformation retract of $|\Delta(P)|-|\Delta(P-Q)|$. 
The order complex of the poset $\mathcal{K}_{\text {link }}\left(\mathcal{A} \otimes \mathbb{R}^{\ell+1}\right)$ can be identified with the intersection of $S\left(V \otimes \mathbb{R}^{\ell+1}\right)$ and the arrangement tensored with $\mathbb{R}^{\ell+1}$.

Lemma 5.33. We have a homeomorphism

$$
\left|\Delta\left(\mathcal{K}_{\text {link }}\left(\mathcal{A} \otimes \mathbb{R}^{\ell+1}\right)\right)\right| \cong S\left(V \otimes \mathbb{R}^{\ell+1}\right) \cap\left(\bigcup_{L \in \mathcal{A}} L \otimes \mathbb{R}^{\ell+1}\right) .
$$

Thus $\operatorname{Sal}^{(\ell)}(\mathcal{A})$ is homotopy equivalent to $V \otimes \mathbb{R}^{\ell+1}-\bigcup_{L \in \mathcal{A}} L \otimes \mathbb{R}^{\ell+1}$ and Theorem 5.29 is proved.

$\mathrm{Sal}^{(\ell)}(\mathcal{A})$ is a simplicial complex as the order complex of a poset. As we have done in $\S 2$, we can glue simplices together to form a regular cell complex with fewer cells. Instead of repeating the same argument, we use the following fact observed in [BZ92].

Proposition 5.34. Let $K$ be a PL regular cell decomposition of a sphere $S^{n}$. For a subcomplex $L$, let $Q$ be the order ideal in the face poset $P$ of $K$. Then there exists a regular cell complex $L^{\prime}$ with the following properties:

1. $L^{\prime}$ is a subcomplex of the opposite regular cell decomposition of $K$,

2. the face poset of $L^{\prime}$ is isomorphic to $(P-Q)^{\mathrm{op}}$,

3. $\left|L^{\prime}\right|$ is homotopy equivalent to $|K|-|L|$.

Thus we can regard $\operatorname{Sal}^{(\ell)}(\mathcal{A})$ as a subcomplex of $S\left(V \otimes \mathbb{R}^{\ell+1}\right)$ under a suitable regular cell decomposition of $S\left(V \otimes \mathbb{R}^{\ell+1}\right)$.

Now consider the braid arrangement $A_{k-1}$. We have

$$
\operatorname{Sal}^{(\ell-1)}\left(\mathcal{A}_{k-1}\right) \simeq \mathfrak{h}_{k} \otimes \mathbb{R}^{\ell}-\bigcup_{1 \leq i<j \leq k} L_{i, j}^{\prime} \otimes \mathbb{R}^{\ell} \simeq F\left(\mathbb{R}^{\ell}, k\right) \simeq \mathcal{C}_{\ell}(k) .
$$

The above homotopy equivalences are all $\Sigma_{k}$-equivariant.

The skeletal filtration on $\mathrm{Sal}^{(\ell-1)}\left(\mathcal{A}_{k-1}\right)$ induces the following spectral sequence.

Theorem 5.35. For any homology theory there exists a spectral sequence

$$
E^{1} \cong \bigoplus_{k} C_{*}\left(\operatorname{Sal}^{(\ell-1)}\left(\mathcal{A}_{k-1}\right)\right) \otimes_{\Sigma_{k}} \tilde{h}_{*}\left(X^{\wedge k}\right) \Rightarrow h_{*}\left(\Omega^{\ell} \Sigma^{\ell} X\right),
$$

which is a direct sum of spectral sequences each of which strongly converges to the corresponding direct summand in (4). In particular, when $h_{*}(-)$ satisfies the strong form of the Künneth formula, the $E^{1}$-term is a functor of $h_{*}(X)$.

Note that in the case $\ell=2$, the $E^{1}$-term is described as a functor of $h_{*}(\Sigma X)$. The shift of degree by the suspension functor $\Sigma$ is used to obtain an isomorphism between the $E^{2}$ term and Cotor. It is natural to expect that, when $\ell>2$, the $E^{2}$-term can also be expressed as a certain derived functor by making appropriate degree shifts in $h_{*}(X)$.

\section{Concluding remarks}

We conclude this paper by making two remarks on relations to other works. 


\subsection{The Arone-Ahearn-Kuhn spectral sequence}

For the singular homology theory $H_{*}(-; k)$ with coefficients in a field $k$, Smirnov [Smi02] constructed a spectral sequence converging to $H_{*}\left(\Omega^{\ell} Z ; k\right)$ whose $E^{1}$-term is a functor of $H_{*}(Z ; k)$.

On the other hand, Ahearn and Kuhn studied the Goodwillie tower of the functor $\Sigma^{\infty} \operatorname{Map}_{*}(K, Z)$, in particular $\Sigma^{\infty} \Omega^{\ell} Z$, in [AK02, Kuh] based on the analysis Arone did in [Aro99]. Kuhn remarks in [Kuh] that, when $K$ is a sphere, the spectral sequence

$$
E^{1}(Z ; \ell) \Rightarrow h_{*}\left(\Omega^{\ell} Z\right)
$$

obtained from the Arone-Goodwillie tower must necessarily agree with Smirnov's.

Arone's model describes the layers in the Arone-Goodwillie tower in terms of little cubes. In particular, when $Z=\Sigma^{\ell} X$, the tower coincides with the Snaith splitting and the spectral sequence collapses at the $E^{1}$-term.

Our spectral sequence constructed in $\$ 5$ is much finer than the Arone-Ahearn-Kuhn spectral sequence (11) for $Z=\Omega^{\ell} X$. It is highly nontrivial. It would be interesting to compare our spectral sequence and the Arone-Ahearn-Kuhn spectral sequence in the case

$$
E^{1}\left(\Omega^{\ell-m} \Sigma^{\ell} X ; m\right) \Rightarrow h_{*}\left(\Omega^{\ell} \Sigma^{\ell} X\right) .
$$

\subsection{Milgram's model and free iterated monoidal categories}

This paper is based on May's little cube model [May72] of the $\ell$-fold loop space freely generated by a based space $X$,

$$
\Omega^{\ell} \Sigma^{\ell} X \simeq\left(\coprod_{k \geq 0} \mathcal{C}_{\ell}(k) \times_{\Sigma_{k}} X^{k}\right) / \sim .
$$

Alternative models for $\Omega^{\ell} \Sigma^{\ell} X$ have been discovered by several people. In particular, Milgram's model [Mil66] is combinatorial in nature and the referee suggested a possible connection with the construction in $\$ 5$.

In fact, we can see there is an intimate relation between the Salvetti complex for the braid arrangement and Milgram's construction if we use a categorical construction developed in [BFSV03] by Balteanu, Fiedorowicz, Schwänzl, and Vogt.

Recall that Milgram used permutohedra $P_{k}$, which is the convex hull of the orbit

$$
\left\{(\sigma(1), \ldots, \sigma(k)) \mid \sigma \in \Sigma_{k}\right\}
$$

of $(1, \ldots, k) \in \mathbb{R}^{k}$ under the action of $\Sigma_{k}$, to build a model for $\Omega^{\ell} \Sigma^{\ell} X$. One of the ways to describe the construction is as follows. Define

$$
J_{k}^{(\ell)}=\left(P_{k}\right)^{\ell-1} \times \Sigma_{k} / \sim
$$

by using a certain equivalence relation $\sim$ gluing the boundary cells. See Berger's article in [Vog] for more details. $J_{k}^{(\ell)}$ is equipped with a right action of $\Sigma_{k}$ by definition. Define

$$
J^{(\ell)}(X)=\left(J_{k}^{(\ell)} \times \Sigma_{k} X^{k}\right) / \sim
$$


where is the "base point relation". It is the main result of [Mil66] that

$$
J^{(\ell)}(X) \simeq \Omega^{\ell} \Sigma^{\ell} X
$$

for a connected pointed CW-complex $X$.

Balteanu, Fiedorowicz, Schwänzl, and Vogt found a categorical model for $J_{k}^{(\ell)}$ in [BFSV03]. They defined the notion of $\ell$-fold monoidal category. They also defined a free $\ell$-fold monoidal category $\mathcal{F}_{\ell}(S)$ generated by a set $S$. Objects are finite expressions generated by elements of $S$ using associative operations $\square_{1}, \ldots, \square_{\ell}$. For example,

$$
\left(i_{1,1} \square_{1} i_{1,2} \square_{1} \cdots \square_{1} i_{1, s_{1}}\right) \square_{2} \cdots \square_{2}\left(i_{\ell-r, 1} \square_{1} \cdots \square_{1} i_{\ell-r, s_{\ell-r}}\right)
$$

is an element of $\mathcal{F}_{2}(S)$, where $i_{1,1}, \ldots, i_{\ell-r, s_{\ell-r}} \in S$. Morphisms are generated by

$$
\eta_{a b c d}^{i j}:\left(a \square_{j} b\right) \square_{i}\left(c \square_{j} d\right) \rightarrow\left(a \square_{i} c\right) \square_{j}\left(b \square_{i} d\right)
$$

for $a, b, c, d \in S$ and $1 \leq i<j \leq \ell$. They studied the full subcategory $\mathcal{M}_{\ell}(k)$ of $\mathcal{F}_{\ell}(\{1, \ldots, k\})$ consisting of expressions in which each element $1, \ldots, k$ appears exactly once. Define a full subcategory $\overline{\mathcal{J}}_{\ell}(k)$ consisting of expressions in which $\square_{1}$ appears in the outermost level, the operation $\square_{2}$ appears in the next level, and so on. For example, (12) is an element of $\overline{\mathcal{J}}_{2}(k)$.

The category $\overline{\mathcal{J}_{\ell}}(k)$ is a poset. Furthermore it is easy to see that it is isomorphic to $\mathcal{L}^{(\ell-1)}\left(\mathcal{A}_{k}\right)$ by using the fact that the elements of the face poset $\mathcal{F}\left(\mathcal{A}_{k}\right)$ of the braid arrangement $\mathcal{A}_{k}$ are given by partitions of $\{1, \ldots, k\}$. The higher order Salvetti complex $\mathrm{Sal}^{(\ell-1)}\left(\mathcal{A}_{k}\right)$ for the braid arrangement can be identified with the order complex of $\overline{\mathcal{J}}_{\ell}(k)$.

Balteanu, Fiedorowicz, Schwänzl, and Vogt compared $\overline{\mathcal{J}}_{\ell}(k)$ and Milgram's construction in $\S 5$ of their paper. They proved that the classifying spaces of the categories $\left\{\overline{\mathcal{J}}_{2}(k)\right\}_{k \geq 0}$ give rise to a construction

$$
\bar{J}_{2}(X)=\left(\coprod_{k \geq 0} B \overline{\mathcal{J}}_{2}(k) \times \Sigma_{\Sigma_{k}} X^{k}\right) / \sim
$$

which is homeomorphic to Milgram's construction $J^{(2)}(X)$ (Theorem 3.12). For $\ell>2$, they noticed that the analogous construction $\bar{J}_{\ell}(X)$ does not coincide with Milgram's construction on the nose, but the natural quotient map

$$
q_{k}: J_{k}^{(\ell)} \rightarrow B \overline{\mathcal{J}}_{\ell}(k)=\operatorname{Sal}^{(\ell-1)}\left(\mathcal{A}_{k}\right)
$$

induces a homotopy equivalence of preoperads (Theorem 3.13). Since $q_{k}$ maps cells onto cells, we can define a cellular filtration on $J_{k}^{(\ell)}$ which coincides with the skeletal filtration on $\mathrm{Sal}^{(\ell-1)}\left(\mathcal{A}_{k}\right)$ up to homotopy. Thus it is possible to construct the spectral sequence in Theorem 5.35 in terms of Milgram's model, i.e. permutohedra.

The author thinks, however, that the skeletal filtration on the higher order Salvetti complex is better in the sense that it is obtained by collapsing "homotopy-theoretically redundant" information from Milgram's construction. In particular, it is better suited for analyzing the spectral sequence. 
Acknowledgments. It was an e-mail message from Ryan Budney which drove the author to try to find a relation between the Salvetti complex and the filtration on little 2-cubes. The author is grateful to him for his intriguing question. The result of this paper partially answers his question. Tilman Bauer kindly informed the author of the inaccuracy of the statement of the main result in [Tam94]. The author would also like to thank the referee for his/her helpful comments, especially for pointing out an overlap with a construction by De Concini and Salvetti in [DCS00] and a possible connection with the work of Milgram [Mil66]. Finally it is a pleasure to acknowledge the interests and suggestions expressed by Fred Cohen and Jim Stasheff which helped the author a lot to improve the earlier versions of this paper.

This research was partially supported by Grants-in-Aid for Scientific Research, Ministry of Education, Culture, Sports, Science and Technology, Japan, no. 17540070.

\section{References}

[AK02] Ahearn, S. T., Kuhn, N. J.: Product and other fine structure in polynomial resolutions of mapping spaces. Algebr. Geom. Topol. 2, 591-647 (2002) Z Zbl 1015.55006 MR 1917068

[Aro99] Arone, G.: A generalization of Snaith-type filtration. Trans. Amer. Math. Soc. 351, 1123-1150 (1999) Zbl 0945.55011 MR 1638238

[Arv91] Arvola, W. A.: Complexified real arrangements of hyperplanes. Manuscripta Math. 71, 295-306 (1991) Zbl 0731.57011 MR 1103735

[BFSV03] Balteanu, C., Fiedorowicz, Z., Schwänzl, R., Vogt, R.: Iterated monoidal categories. Adv. Math. 176, 277-349 (2003) Zbl 1030.18006 MR 1982884

[BLVS $\left.{ }^{+} 99\right]$ Björner, A., Las Vergnas, M., Sturmfels, B., White, N., Ziegler, G. M.: Oriented Matroids. Encyclopedia Math. Appl. 46, Cambridge Univ. Press, Cambridge (1999) Zbl 0773.52001 MR 1744046

[BZ92] Björner, A., Ziegler, G. M.: Combinatorial stratification of complex arrangements. J. Amer. Math. Soc. 5, 105-149 (1992) Zbl 0754.52003 MR 1119198

[DCS00] De Concini, C., Salvetti, M.: Cohomology of Coxeter groups and Artin groups. Math. Res. Lett. 7, 213-232 (2000) Zbl 0972.20030 MR 1764318

[EM66a] Eilenberg, S., Moore, J. C.: Homological algebra and fibrations. In: Colloque de Topologie (Brussels, 1964), Librairie Universitaire, Louvain, 81-90 (1966) Zbl 0138.18205 MR 0218425

[EM66b] Eilenberg, S., Moore, J. C.: Homology and fibrations. I. Coalgebras, cotensor product and its derived functors. Comment. Math. Helv. 40, 199-236 (1966) Zbl 0148.43203 MR 0203730

[GR89] Gel'fand, I. M., Rybnikov, G. L.: Algebraic and topological invariants of oriented matroids. Dokl. Akad. Nauk SSSR 307, 791-795 (1989) (in Russian) Z Zbl 0717.33009 MR 1020668

[Kuh] Kuhn, N. J.: Topological nonrealization results via the Goodwillie tower approach to iterated loopspace homology, Algebr. Geom. Topol. 8, 2109-2129 (2008); correction ibid. 10, 531-533 (2010) Zbl 1169.55011 MR 2460881

[May72] May, J. P.: The Geometry of Iterated Loop Spaces. Springer, Berlin (1972) Zbl 0244.55009 MR 0420610

[Mil66] Milgram, R. J.: Iterated loop spaces. Ann. of Math. (2) 84, 386-403 (1966) Zbl 0145.19901 MR 0206951

[Rec70] Rector, D. L.: Steenrod operations in the Eilenberg-Moore spectral sequence. Comment. Math. Helv. 45, 540-552 (1970) Zbl 0209.27501 MR 0278310 
[Sal87] Salvetti, M.: Topology of the complement of real hyperplanes in $\mathbf{C}^{N}$. Invent. Math. 88, 603-618 (1987) Zbl 0594.57009 MR 0884802

[Smi02] Smirnov, V. A.: The homology of iterated loop spaces. Forum Math. 14, 345-381 (2002) Zbl 1026.55013 MR 1899289

[Sna74] Snaith, V. P.: A stable decomposition of $\Omega^{n} S^{n} X$. J. London Math. Soc. (2) 7, 577-583 (1974) Zbl 0275.55019 MR 0339155

[Tam94] Tamaki, D.: A dual Rothenberg-Steenrod spectral sequence. Topology 33, 631-662 (1994) Zbl 0820.55009 MR 1293304

[Tam07] Tamaki, D.: On the $E^{1}$-term of the gravity spectral sequence. In: Proceedings of the Nishida Fest (Kinosaki, 2003), Geom. Topol. Monogr. 10, 347-382 (2007) MR 2402794

[Vog] Vogt, R.: Workshop on Operads,

http://www.mathematik.uni-bielefeld.de/sfb343/preprints/pre99005.ps.gz 